\title{
Reciprocal regulation of LOXL2 and HIF1a drives the Warburg effect to support pancreatic cancer aggressiveness
}

\author{
Rongkun $\mathrm{Li}^{1,2,7}$, Hengchao $\mathrm{Li}^{3,7}$, Lili Zhu ${ }^{2,7}$, Xiaoxin Zhang ${ }^{4,7}$, Dejun Liu ${ }^{5}$, Qing Li ${ }^{2}{ }^{2}$, Bo $\mathrm{Ni}^{6}$, Lipeng Hu${ }^{2}$, Zhigang Zhang ${ }^{2}$,

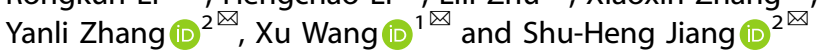

(c) The Author(s) 2021

Hypoxic microenvironment is common in solid tumors, particularly in pancreatic ductal adenocarcinoma (PDAC). The Warburg effect is known to facilitate cancer aggressiveness and has long been linked to hypoxia, yet the underlying mechanism remains largely unknown. In this study, we identify that lysyl oxidase-like 2 (LOXL2) is a hypoxia-responsive gene and is essential for the Warburg effect in PDAC. LOXL2 stabilizes hypoxia-inducible factor 1a (HIF1a) from prolyl hydroxylase (PHD)-dependent hydroxylation via hydrogen peroxide generation, thereby facilitating the transcription of multiple glycolytic genes. Therefore, a positive feedback loop exists between LOXL2 and HIF1a that facilitates glycolytic metabolism under hypoxia. Moreover, LOXL2 couples the Warburg effect to tumor growth and metastasis in PDAC. Hijacking glycolysis largely compromises LOXL2-induced oncogenic activities. Collectively, our results identify a hitherto unknown hypoxia-LOXL2-HIF1a axis in regulating the Warburg effect and provide an intriguing drug target for PDAC therapy.

Cell Death and Disease (2021)12:1106; https://doi.org/10.1038/s41419-021-04391-3

\section{BACKGROUND}

Pancreatic ductal adenocarcinoma (PDAC) is one of the most intractable and lethal cancers and has been the seventh leading cause of cancer-related deaths worldwide [1, 2]. Unfortunately, despite the improvements in diagnostic and therapeutic strategies for PDAC, the prognosis is still dismal reflected by a 5 -year overall survival rate around of $8 \%$ [3]. Thus, there is a great need for further mechanistic understanding that triggers PDAC progression to be used for more effective therapeutic approaches for the treatment of PDAC.

Hypoxic microenvironment is a common feature of solid tumors and is particularly notable in PDAC due to poor blood flow caused by the desmoplastic reaction $[4,5]$. To cope with hypoxia, cancer cells have developed numerous adaptive responses, during which hypoxia-inducible factor 1 (HIF1) plays central roles by activating a host of hypoxia-responsive genes [5, 6]. HIF1 is comprised of HIF1a and HIF1 $\beta$, wherein HIF1a serves as the major regulatory subunit responsible for its transcriptional function [7]. Both the stability and activity of HIF1a are oxygen-dependently regulated. Under normoxic conditions, HIF1a is hydroxylated by oxygen-dependent prolyl hydroxylases (PHDs), which enable the tumor suppressor von Hippel-Lindau (VHL) to bind to and mark HIF1a for rapid degradation through the ubiquitin-proteasome pathway. Under hypoxic conditions, prolyl hydroxylation of HIF1a is blocked, leading to HIF1a stabilization and nuclear translocation $[6,8]$. In the nucleus, HIF1a dimerizes with HIF1 $\beta$ and binds to the hypoxia response elements (HREs) in the promoter regions of target genes involved in a plethora of pathophysiological processes.

Under hypoxic conditions, cancer cells are required to meet their oxygen demand by reprogramming their metabolic pathways, such as switching glycolytic reprogramming from oxidative phosphorylation to glycolysis [9]. Cancer cells exhibit aberrant metabolism characterized by high glycolysis even with sufficient oxygen, a phenomenon known as aerobic glycolysis or the Warburg effect $[10,11]$. Such metabolic reprogramming not only improves cancer cell adaption potential to fluctuating oxygen tension, but also provides cancer cells with a proliferation advantage by producing ATP as well as glycolytic intermediates for biosynthesis of nucleotides, lipids, and proteins [10]. Simultaneously, through this metabolic alteration, more than $90 \%$ of glucose is converted to lactate, which facilitates acidification of the tumor microenvironment to favor tumor invasion and metastasis $[12,13]$.

Lysyl oxidase-like 2 (LOXL2) is a member of the lysyl oxidase (LOX) family, which are copper- and quinone-dependent amine oxidases that promote the cross-linking of collagen and elastin, major components of desmoplastic stroma and fibrosis [14]. The LOX family is constituted by five members, LOX and lysyl oxidaselike 1-4 (LOXL1-4), all of which share a highly conserved enzymatic domain in C-terminus required for the oxidative deamination of

\footnotetext{
${ }^{1}$ Institute of Oncology, Affiliated Hospital of Jiangsu University, Zhenjiang 212001, China. ${ }^{2}$ State Key Laboratory of Oncogenes and Related Genes, Shanghai Cancer Institute, Ren Ji Hospital, School of Medicine, Shanghai Jiao Tong University, Shanghai 200240, China. ${ }^{3}$ Department of Pancreatic surgery, Huashan Hospital, Fudan University, Shanghai 200040, China. ${ }^{4}$ Jiangsu Key Laboratory of Medical Science and Laboratory Medicine, School of Medicine, Jiangsu University, Zhenjiang 212013 , China. ${ }^{5}$ Department of BiliaryPancreatic Surgery, Ren Ji Hospital, School of Medicine, Shanghai Jiao Tong University, Shanghai 200127, China. ${ }^{6}$ Department of Gastrointestinal Surgery, Ren Ji Hospital, School of Medicine, Shanghai Jiao Tong University, Shanghai 200127, China. ${ }^{7}$ These authors contributed equally: Rongkun Li, Hengchao Li, Lili Zhu, Xiaoxin Zhang. 冈email: ylzhang@shsci.org; wangxu@ujs.edu.cn; shjiang@shsci.org

Edited by Jean-Ehrland Ricci
}

Received: 22 June 2021 Revised: 2 November 2021 Accepted: 10 November 2021

Published online: 26 November 2021 
peptidyl-lysine residues in substrates, generating an aldehyde group and a byproduct, hydrogen peroxide [15]. Accumulated studies have documented the roles of LOXL2 in tumorigenesis [16], however, the relationship between LOXL2 and cancer metabolism remains unclear.

Here, we described that LOXL2, induced by hypoxia, couples the Warburg effect to tumor progression in PDAC. Mechanistically, LOXL2 stabilizes HIF1a by inhibiting HIF1a hydroxylation dependent on its catalytic activity through a hydrogen peroxidemediated mechanism and enhances the expression of HIF1a target genes, thereby promoting aerobic glycolysis and PDAC progression. The results strongly suggest that the LOXL2-HIF1a positive feedback loop might be a potential target for PDAC cancer therapy.

\section{RESULTS}

Overexpressed LOXL2 predicts poor prognosis in PDAC

To investigate the LOX family members in PDAC, we first analyzed their expression pattern in PDAC and normal pancreas tissues using Gene Expression Omnibus (GEO) datasets. As a result, LOXL2 mRNA level in PDAC was significantly higher than that in normal pancreas as shown in Fig. $1 \mathrm{~A}$ and Fig. S1A-D. So, we selected LOXL2 for further study in PDAC. Consistently, a significantly higher LOXL2 expression was observed in Ren Ji cohort (GSE102238) (Fig. 1B). IHC analysis of LOXL2 in a tissue microarray revealed that high LOXL2 expression was observed in the majority of PDAC tissues but not in the non-tumor tissues (Fig. 1C, D). Moreover, LOXL2 expression was also remarkably upregulated in PDAC tissues of $\mathrm{Kras}^{\mathrm{G} 12 \mathrm{D} /+}$; $\operatorname{Trp} 53^{\mathrm{R} 172 \mathrm{H} /+}$; Pdx1-Cre (KPC) mice as compared with normal pancreas (Fig. 1E). Of note, LOXL2 staining was mainly distributed in the nuclear and cytoplasm of PDAC cells (Fig. 1C, E).

To elucidate the clinical relevance, we analyzed the association between LOXL2 expression and the clinicopathologic features of PDAC patients. High LOXL2 expression was associated with more aggressive tumor behaviors, including larger tumor size, advanced T stage, and frequent distant metastasis (Table S1). Additionally, Kaplan-Meier curves showed that the overall survival times were shorter in patients with high LOXL2 than those with low LOXL2 expression (Fig. 1F), which was further supported by the survival analyses of The Cancer Genome Atlas (TCGA) and GEO (GSE57495 and GSE62452) cohorts (Fig. 1G-I). Univariate and multivariate analyses revealed that LOXL2 expression was an independent predictor for the overall survival in addition to tumor size, tumor differentiation, and lymph node metastasis (Fig. 1J, K). Taken together, these findings suggest that LOXL2 is commonly upregulated and may serve as a predictor of malignant progression in PDAC.

\section{Hypoxia induces LOXL2 expression of PDAC cells}

To pursue the mechanism of LOXL2 dysregulation in PDAC, we tested whether LOXL2 expression could be induced by hypoxia. In four PDAC cells exposed to hypoxia for up to $48 \mathrm{~h}$, LOXL2 was found to increase in hypoxia compared to normoxia at both mRNA (Fig. 2A) and protein (Fig. 2B) levels. Then, siRNAs targeting HIF1a were used to confirm whether LOXL2 expression induced by hypoxia is dependent on HIF1a in PDAC cells. As a result, HIF1a depletion drastically inhibited LOXL2 expression at both mRNA (Fig. 2C) and protein (Fig. 2D) levels during both normoxic and hypoxic conditions. Therefore, LOXL2 is induced by hypoxia in a HIF1a-dependent fashion. To determine whether LOXL2 and HIF1a are clinically pertinent, we analyzed the TCGA cohort for the expression of LOXL2 and a 15-gene hypoxia signature that reflects hypoxia status [17]. There was a significantly positive correlation between LOXL2 and the hypoxia signature (Fig. 2E), which was supported by the results of GSEA analyses using public GEO and TCGA databases that gene signatures related to hypoxia were significantly enriched in LOXL2-high samples compared with LOXL2-low samples (Fig. 2F). Furthermore, a high 15-gene hypoxia signature was associated with worse overall survival and diseasefree survival in PDAC (Fig. 2G). These findings suggest that LOXL2 is a hypoxia-responsive gene aberrantly expressed in PDAC.

\section{LOXL2 promotes oncogenic progression in PDAC}

To determine the involvement of LOXL2 in the progression of PDAC, we first performed loss-of-function studies in two cell lines (Patu8988 and MIA PaCa-2) with higher LOXL2 expression (Fig. S2A, B). Two specific shRNAs against LOXL2 led to marked downregulation of LOXL2 as revealed by $\mathrm{qRT}-\mathrm{PCR}$ and western blotting (Fig. S2C, D). LOXL2 knockdown impaired PDAC cell proliferation and colony formation ability (Fig. 3A, B). Likewise, LOXL2 knockdown resulted in significantly reduced numbers of migrated cells (Fig. 3C) and invaded cells (Fig. 3D). Next, we established two LOXL2 overexpression cell models in AsPC-1 and SW1990 cells, which had lower endogenous LOXL2 expression (Fig. S2A, B and S2E, F). As expected, an opposite phenomenon was noticed in gain-of-function studies (Fig. 3E-H). To explore whether the in vitro findings could be recapitulated in vivo, we generated subcutaneous xenografts and an intrasplenic implantation mouse model. Compared to xenografts from the control group, LOXL2 knockdown robustly repressed tumor growth (Fig. 4A, B), which was further confirmed by IHC staining of the proliferation index PCNA (Fig. 4C). Furthermore, liver metastasis lesions were preferentially reduced with LOXL2 loss (Fig. 4G, H). Correspondingly, increased tumor growth (Fig. 4D-F) and liver metastasis (Fig. $4 \mathrm{l}, \mathrm{J}$ ) were obtained after LOXL2 overexpression. Altogether, these results suggest that LOXL2 confers growth and metastasis advantages to PDAC cells.

\section{LOXL2 enhances aerobic glycolysis of PDAC cells}

To identify the mechanism by which LOXL2 promotes PDAC progression, we performed GSEA using public GEO and TCGA databases. As a result, gene signatures related to glycolysis were significantly enriched in LOXL2-high samples compared with LOXL2-low samples (Fig. 5A). Coincidentally, the medium culture of LOXL2 knockdown cells turned to be acidic much slower and later than that of control cells, especially under hypoxic conditions (Fig. S3A). These findings prompted us to speculate that LOXL2 might exert a role in regulating aerobic glycolysis in PDAC. The notion that LOXL2 regulates the Warburg metabolism of PDAC cells is supported by the results that (i) genetic manipulation of LOXL2 robustly affected the expression of glucose transporters and many glycolytic components at both mRNA and protein levels (Fig. 5B-E); (ii) LOXL2 knockdown attenuated glucose uptake, lactate production (Fig. 5F), and ECAR (an indicator for glycolysis) (Fig. $5 \mathrm{H}$ ), while LOXL2 overexpression had the opposite effects (Fig. 5G, I). However, LOXL2 knockdown or overexpression slightly affected OCR (an indicator for oxidative phosphorylation) (Fig. S3B, C) and (iii) in a cohort of 25 PDAC patients who received preoperative ${ }^{18} \mathrm{~F}$-fluorodeoxyglucose $\left({ }^{18} \mathrm{~F}-\mathrm{FDG}\right) \mathrm{PET} / \mathrm{CT}$, the maximum standardized uptake value (SUVmax), reflecting metabolic activity, was significantly higher in samples with high LOXL2 expression than that in samples with low LOXL2 expression (Fig. 5J).

Moreover, we grew cells in medium containing galactose instead of glucose, thereby reducing glycolytic flux and forcing the cells to rely on mitochondrial oxidative phosphorylation. Under this condition, LOXL2-overexpressing and vector control cells grew, migrated, and invaded at almost the same rate (Fig. S4A-D), indicating that inhibition of the Warburg effect greatly impeded the ability of LOXL2 to promote tumorigenesis. Likewise, we further treated the cells with 2-deoxy-D-glucose (2-DG), a glycolytic inhibitor, to block the glycolytic pathway. Notably, 2-DG also compromised the oncogenic activities of LOXL2 (Fig. S4E-H). Collectively, these data suggest that increased glycolysis is 
A

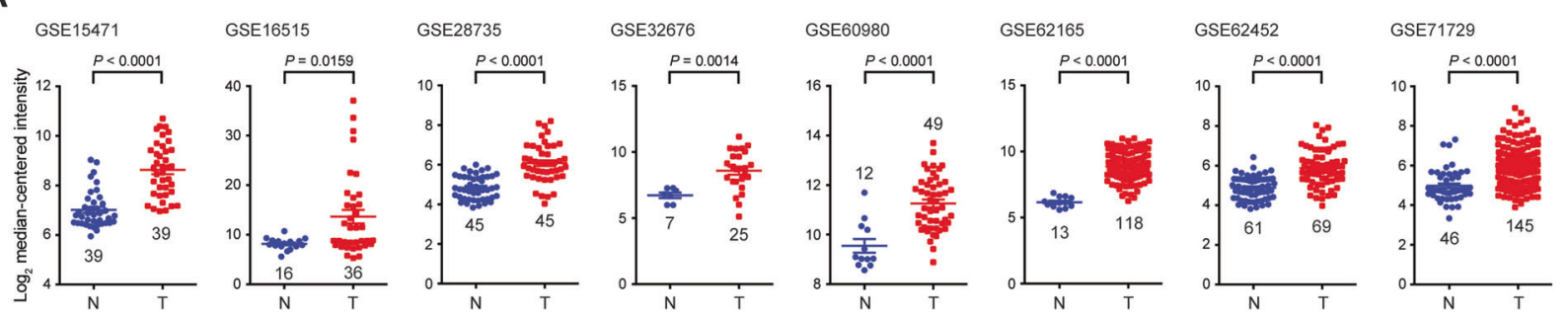

B

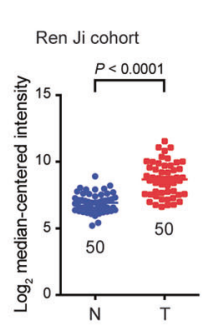

C

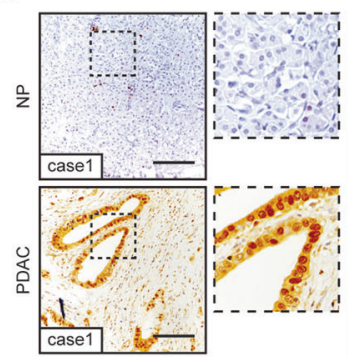

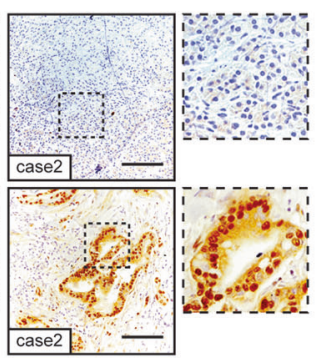

D

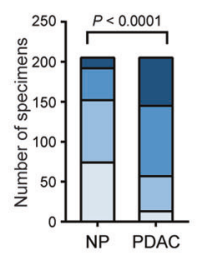

E

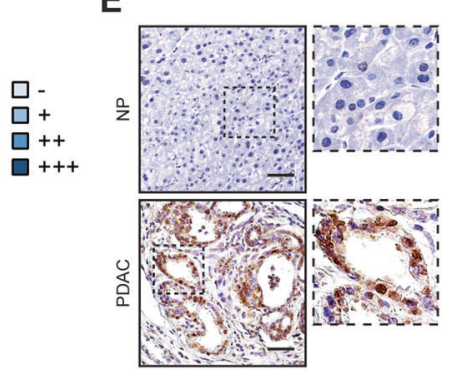

F

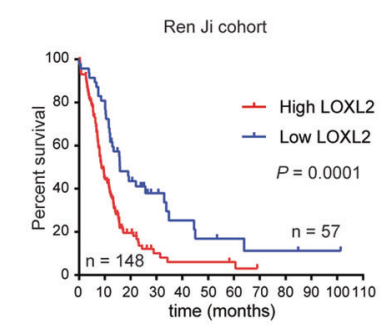

H

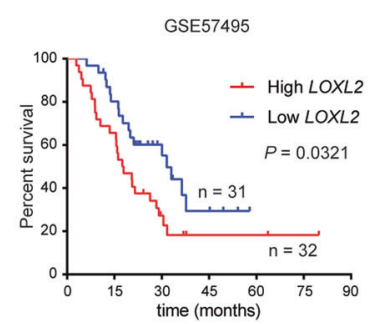

G

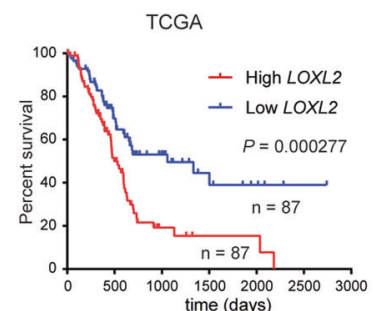

I

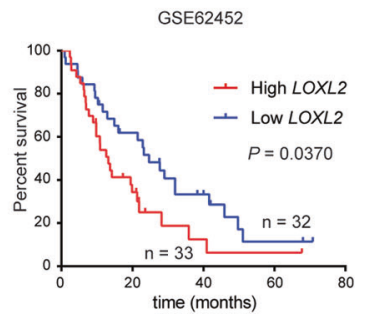

J \begin{tabular}{ll}
$\mathrm{HR}(95 \% \mathrm{Cl})$ & $P$ \\
\hline $1.787(1.165-2.741)$ & $0.008^{\star *}$ \\
$0.772(0.534-1.117)$ & 0.170 \\
$1.311(0.878-1.958)$ & 0.186 \\
$1.499(1.012-2.219)$ & $0.043^{\star}$ \\
$1.655(1.159-2.391)$ & $0.006^{\star \star}$ \\
$0.906(0.553-1.482)$ & 0.693 \\
$1.509(1.065-2.360)$ & $0.023^{\star}$ \\
$0.812(0.426-1.548)$ & 0.526 \\
$1.368(0.548-3.203)$ & 0.470 \\
&
\end{tabular}

K

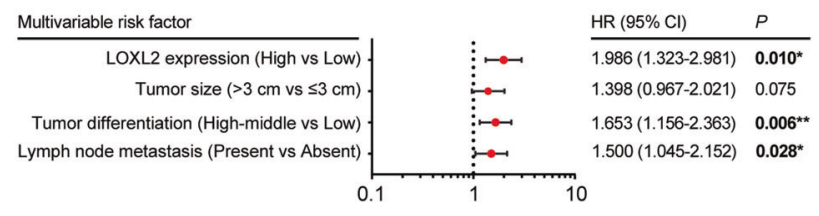

Fig. 1 Expression pattern and prognostic value of LOXL2 in PDAC tissues. A LOXL2 mRNA expression in PDAC tissues (T) and normal pancreas tissues (N) by analyzing multiple gene expression profiles from GEO database. B LOXL2 mRNA expression in PDAC tissues (T) and normal pancreas tissues (N) from Ren Ji hospital published in GSE102238. C LOXL2 expression in the adjacent normal pancreas (NP) and PDAC tissues in tissue microarrays revealed by IHC staining and representative IHC images of LOXL2 in NP and PDAC tissues. Scale bar: $50 \mu \mathrm{m}$. D The percentage of tissue cores displaying no $(-)$, weak $(+)$, moderate $(++)$, or strong $(+++)$ IHC staining of LOXL2 in NP and PDAC tissues. E Representative IHC images of LOXL2 staining in NP and PDAC tissues from Kras ${ }^{\mathrm{TPD} /+}$; Trp53 ${ }^{\mathrm{R} 172 \mathrm{H} /+}+\mathrm{Pdx} 1-\mathrm{Cre}$; (KPC) mice. Scale bar: $50 \mu \mathrm{mm}$. F Comparison of overall survival of PDAC patients with different LOXL2 expression in Ren Ji cohort, G TCGA cohort, H GSE57495 cohort, and I GSE62452 cohort. Survival curves were calculated via the Kaplan-Meier method and analyzed by the log-rank test. J Univariate and K Multivariate analyses showing the association between LOXL2 expression and PDAC survival. CI Confidence interval, HR Hazard ratio. ${ }^{*} p<0.05,{ }^{* *} p<0.01$.

required for LOXL2 to facilitate PDAC progression and can be targeted by glycolysis inhibition.

\section{LOXL2 stabilizes HIF1a via inhibiting its PHD-dependent hydroxylation}

HIF1a is a key transcriptional factor for the Warburg effect $[7,18]$. Considering that multiple glycolytic enzymes regulated by LOXL2 are important transcriptional targets of HIF1a, we hypothesized that LOXL2 was involved in aerobic glycolysis via HIF1a. As expected, under both normoxic and hypoxic conditions, HIF1a protein levels were significantly reduced following LOXL2 knockdown (Fig. 6A), whereas increased following LOXL2 overexpression (Fig. 6B). HIF1a mRNA levels were not affected by LOXL2, suggesting that LOXL2-mediated regulation of HIF1a was translational or posttranslational (Fig. S5A, B), which was supported by the result from analysis of GSE35600 (Fig. S5C). Next, cells were exposed to hypoxia to stabilize HIF1a protein and then treated with cycloheximide (CHX) to arrest protein synthesis. Notably, LOXL2 knockdown shortened the half-life periods of HIF1a under hypoxic conditions (Fig. 6C, D), whereas HIF1a protein degraded at a slower rate after LOXL2 overexpression (Fig. 6E, F), indicating that LOXL2 stabilizes HIF1a. Then, MG132, a proteasome inhibitor, was utilized to prove that the effect of LOXL2 on HIF1a stability was dependent on proteasome-mediated degradation. MG132 treatment abolished altered HIF1a protein stabilization induced by LOXL2 knockdown (Fig. 6G) or overexpression (Fig. 6H). These 
A
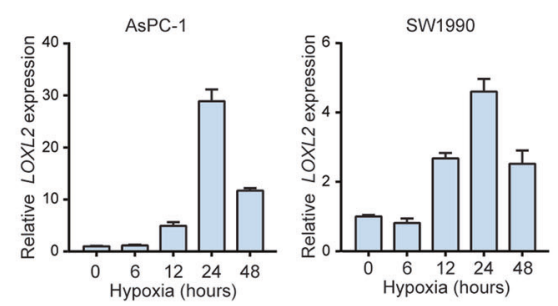

C
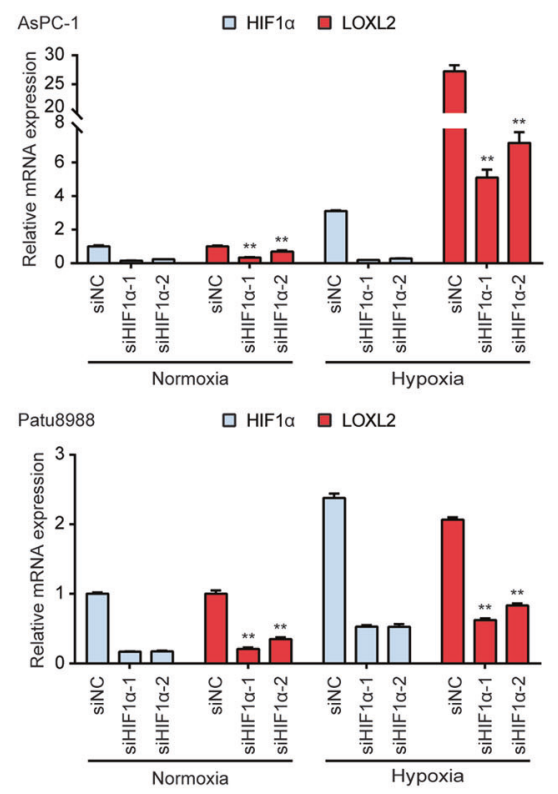

G
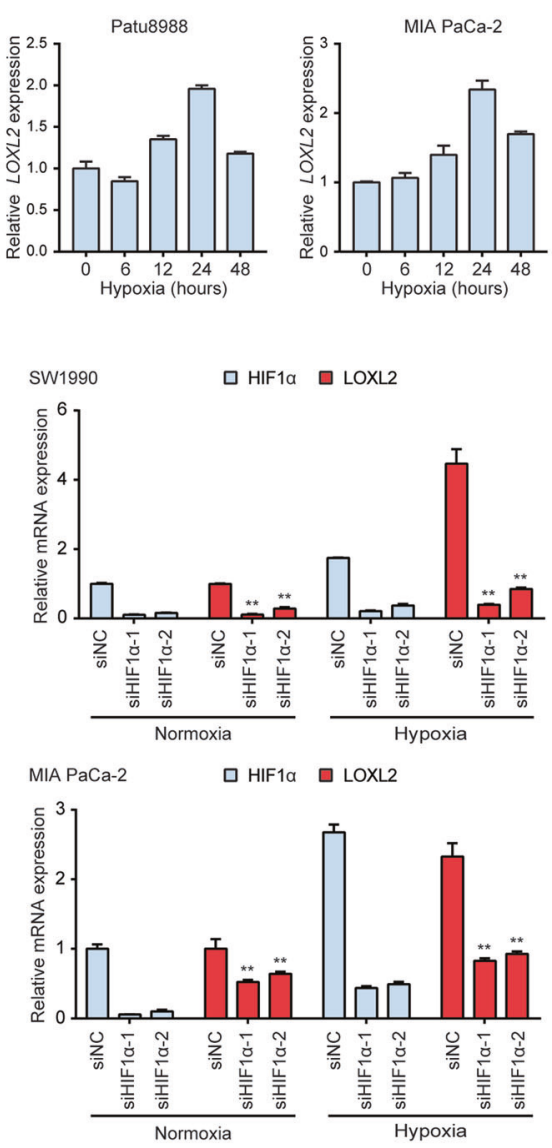

D
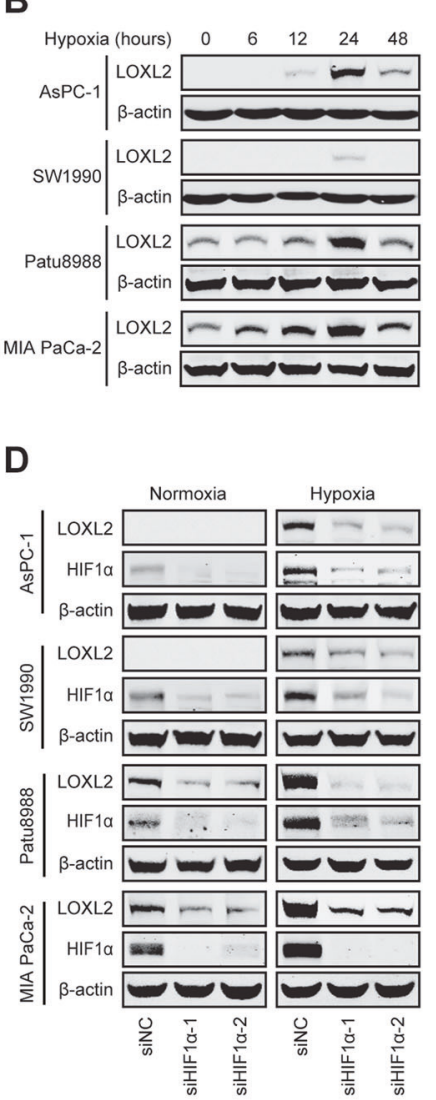

E
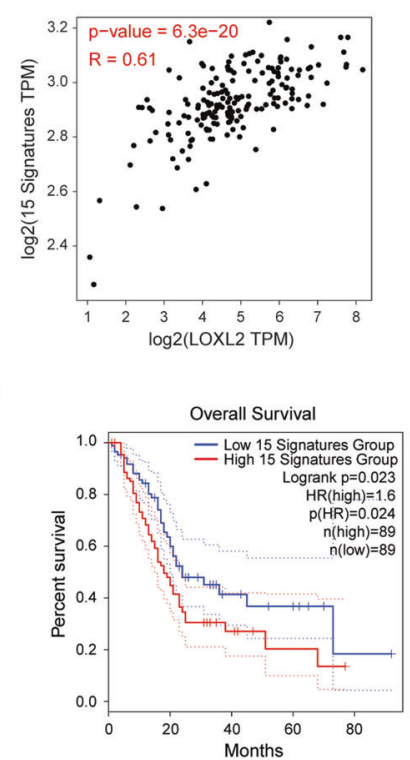

F

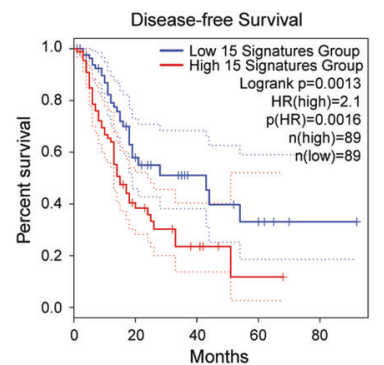

Fig. 2 LOXL2 is induced by hypoxia in PDAC. A LOXL2 mRNA expression in PDAC cells used for LOXL2 overexpression and knockdown exposed to hypoxia for 0-48 h examined by qRT-PCR. B LOXL2 protein expression in PDAC cells used for LOXL2 overexpression or knockdown exposed to hypoxia for $0-48 \mathrm{~h}$ examined by western blotting. C, D The expression of LOXL2 and HIF $1 \alpha$ at mRNA (C) and protein (D) levels in the indicated cells transiently transfected with siRNAs targeting HIF1 $\alpha$ exposed to normoxia or hypoxia for $24 \mathrm{~h}$. E Correlation between LOXL2 expression and the 15-gene hypoxia signature in the PDAC samples of TCGA cohort. F Overall survival and disease-free survival analyses of PDAC patients with different hypoxia status in PDAC samples of TCGA cohort. G Positive correlation between LOXL2 expression and gene set HALLMARK_HYPOXIA in PDAC samples analyzed by GSEA based on the data from GEO and TCGA databases. ${ }^{*} p<0.05,{ }^{* *} p<0.01$. 
A

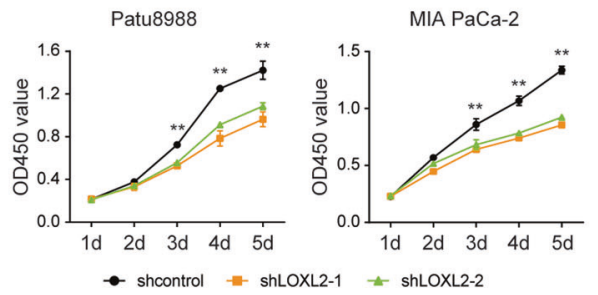

C

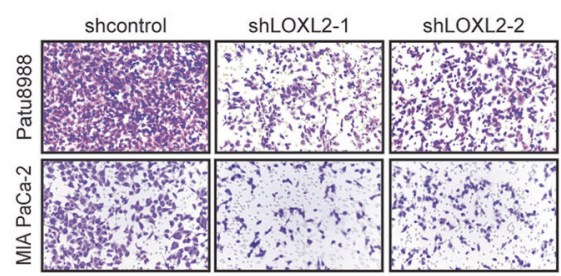

D
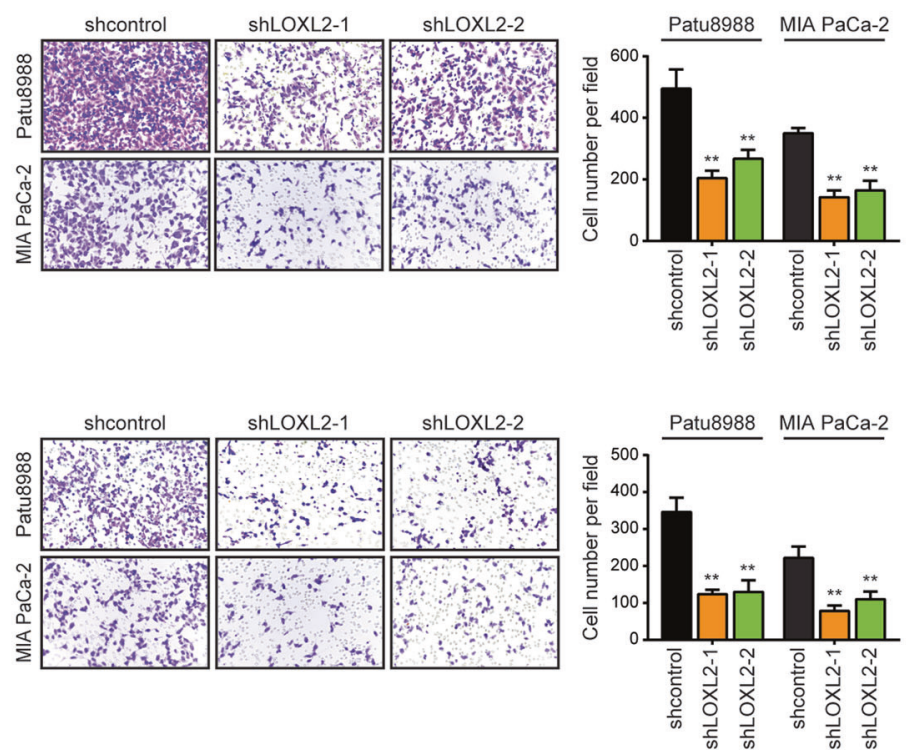

B
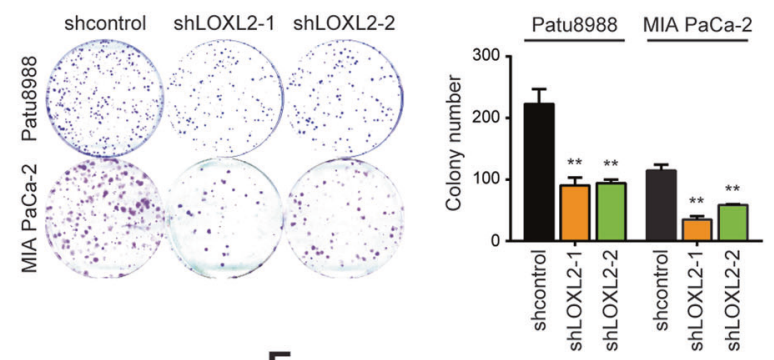

E
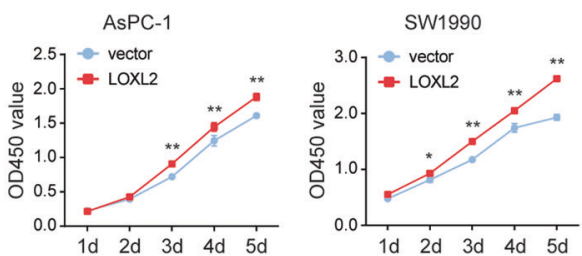

F

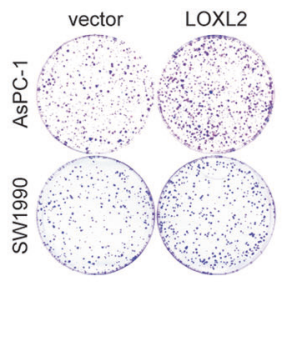

G

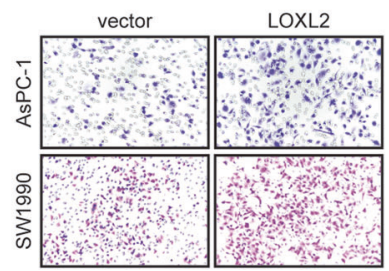

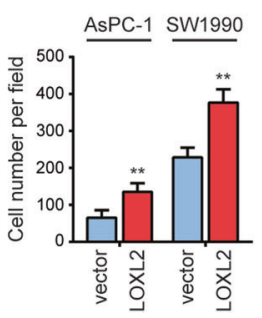

H

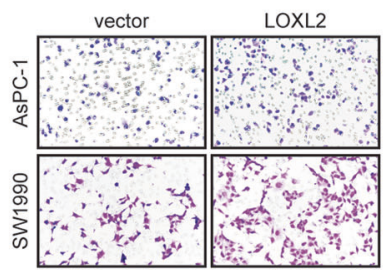

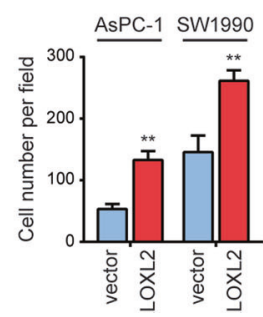

Fig. 3 LOXL2 promotes PDAC cell proliferation, migration, and invasion in vitro. A CCK-8 assay of LOXL2 knockdown and control cells. B Colony formation assay of LOXL2 knockdown and control cells. C Transwell migration assay of LOXL2 knockdown and control cells. D Transwell invasion assay of LOXL2 knockdown and control cells. E CCK-8 assay of LOXL2-overexpressing and vector control cells. F Colony formation assay of LOXL2-overexpressing and vector control cells. G Transwell migration assay of LOXL2-overexpressing and vector control cells. $\mathbf{H}$ Transwell invasion assay of LOXL2-overexpressing and vector control cells. ${ }^{*} p<0.05,{ }^{* *} p<0.01$.

results suggest that LOXL2 exerts a posttranslational regulatory effect on HIF1a protein stability, but not HIF1a protein translation. Next, we explored how LOXL2 regulates the stability of HIF1a protein. LOXL2 protein was observed intensively expressed in the nuclei of PDAC cells (Fig. S6A) and tissues (Fig. 1C and E), and HIF1a functions as a nuclear transcription factor, we therefore postulated a direct interaction between these two proteins because of their similar cellular localization. However, we failed to observe a physical interaction of LOXL2 with HIF1a (Fig. S6B, C), preliminarily ruling out the possibility that LOXL2 stabilizes HIF1a via direct interaction.

Given that lots of oncogenes have been shown to stabilize HIF1a protein via inhibiting its hydroxylation [19-21], we tested whether LOXL2 stabilizes HIF1a by affecting its prolyl hydroxylation. To measure hydroxylated HIF1a levels, cells used for LOXL2 overexpression or knockdown were pretreated with MG132 to prevent hydroxylated HIF1a from being degraded. Less HIF1a but significantly more hydroxylated HIF1a was accumulated during MG132 treatment following LOXL2 knockdown (Fig. 7A), whereas the opposite effects were observed following LOXL2 overexpression (Fig. 7B), suggesting low HIF1a hydroxylation in the presence of LOXL2. HIF1a is hydroxylated on two proline residues (Pro402 and Pro564 in human HIF1a) by a family of oxygen-dependent prolyl hydroxylases (PHD1-4) [6, 8]. Therefore, we asked whether LOXL2 regulates HIF1a hydroxylation through PHD-dependent mechanism. It was validated by determination of the effect of treatment with dimethyloxalylglycine (DMOG), a potent PHD inhibitor, on HIF1a protein stability. If LOXL2 affects HIF1a stability by modulating PHD activity, DMOG treatment would overcome the effects of the manipulation of LOXL2 expression and produce equivalent levels of HIF1a. Indeed, equal levels of HIF1a were observed in response to DMOG treatment in the control and 
A

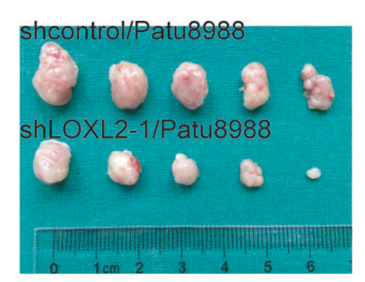

D

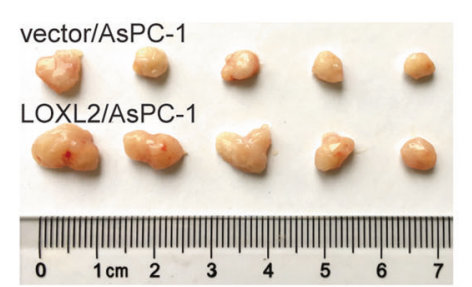

G

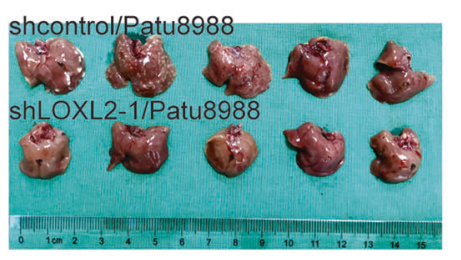

I

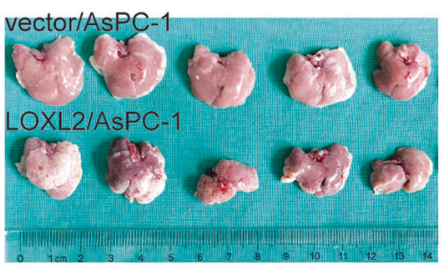

B

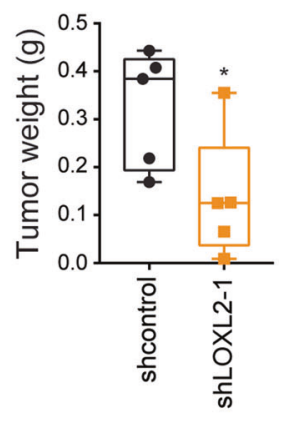

E

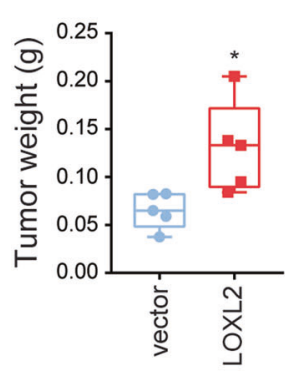

H

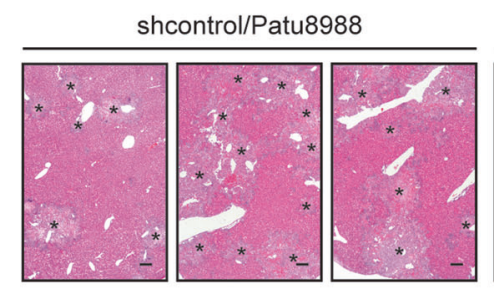

J

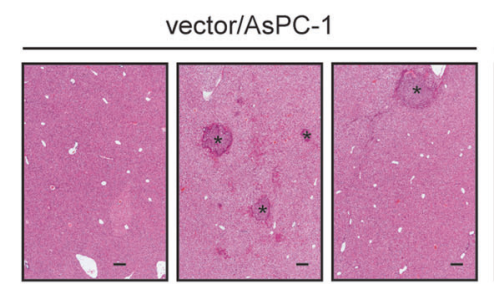

C

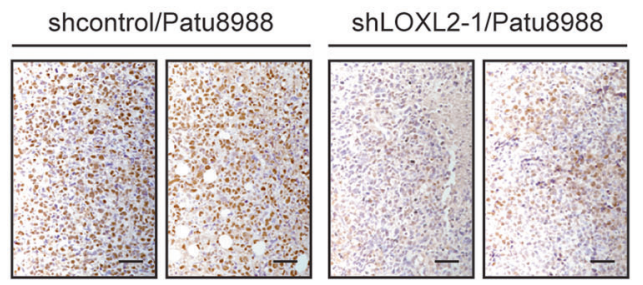

$\mathbf{F}$

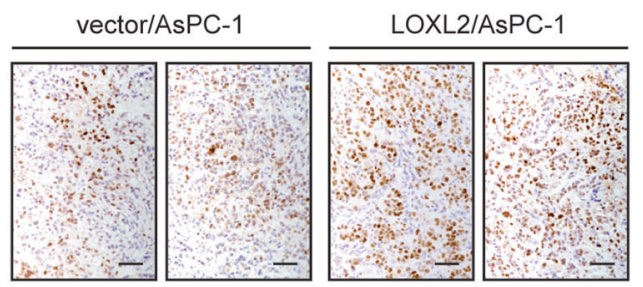

Fig. 4 LOXL2 facilitates tumor growth and liver metastasis of PDAC in vivo. A Growth of subcutaneous xenografts from LOXL2 knockdown and control Patu8988 cells. B Weight of primary subcutaneous tumors of mice in (A). C Representative IHC images of PCNA staining in LOXL2 knockdown and control subcutaneous xenograft tissues. Scale bar: $50 \mu \mathrm{m}$. D Growth of subcutaneous xenografts from LOXL2-overexpressing and vector control AsPC-1 cells. E Weight of primary subcutaneous tumors of mice in (D). F Representative IHC images of PCNA staining in LOXL2-overexpressing and vector control subcutaneous xenograft tissues. Scale bar: $50 \mu \mathrm{m}$. (G) Liver metastasis of intrasplenic xenografts from LOXL2 knockdown and control Patu8988 cells. H Representative hematoxylin and eosin (H\&E) images of liver metastasis in (G). The asterisks indicate liver metastasis lesions. Scale bar: $200 \mu \mathrm{m}$. I Liver metastasis of intrasplenic xenografts from LOXL2-overexpressing and vector control AsPC-1 cells. J Representative H\&E images of liver metastasis in (I). The asterisks indicate liver metastasis lesions. Scale bar: $200 \mu \mathrm{m} .{ }^{*} p<0.05$.

LOXL2 knockdown cells (Fig. 7C), as well as in the vector control and LOXL2-overexpressing cells (Fig. 7D). Overall, these results indicate that LOXL2 reduces PHD activity, and thus, impedes hydroxylation and enhances the stability of HIF1a protein.

\section{LOXL2 catalytic activity is indispensable for its functions in PDAC}

In addition to intracellular oxygen concentration, the activity of PHDs can also be regulated by several intracellular signals, including reactive oxygen species (ROS), which have been shown to inhibit the PHDs and stabilize HIF1a [22]. Hydrogen peroxide, an important member of ROS, is the byproduct of LOXL2 catalytic reaction. We, therefore, reasoned that HIF1a stabilization mediated by LOXL2 is dependent on hydrogen peroxide generation. To test this hypothesis, we generated two LOXL2 mutants affecting the conserved catalytic domain as described previously (Fig. 8A) [23, 24], which was applied to transfect AsPC-1 and SW1990 cells to clarify the implication of LOXL2 enzymatic activity on its action (Fig. 8B). LOXL2 enzymatic activity was subsequently determined by measurement of hydrogen peroxide production. As expected, LOXL2-overexpressing cells had an obvious increase in LOX catalytic activity compared with vector 
A

\begin{tabular}{llll}
\hline Datasets & NES & Nominal p-value & FDR q-value \\
\hline GSE15471 & 1.6548 & 0.0099 & 0.0653 \\
GSE16515 & 1.7912 & 0.0000 & 0.0428 \\
GSE28735 & 1.8679 & 0.0000 & 0.0703 \\
GSE60980 & 1.5535 & 0.0247 & 0.1729 \\
GSE62165 & 1.7777 & 0.0000 & 0.1397 \\
GSE62452 & 1.7005 & 0.0104 & 0.0790 \\
TCGA & 2.1482 & 0.0000 & 0.0000 \\
\hline
\end{tabular}

B
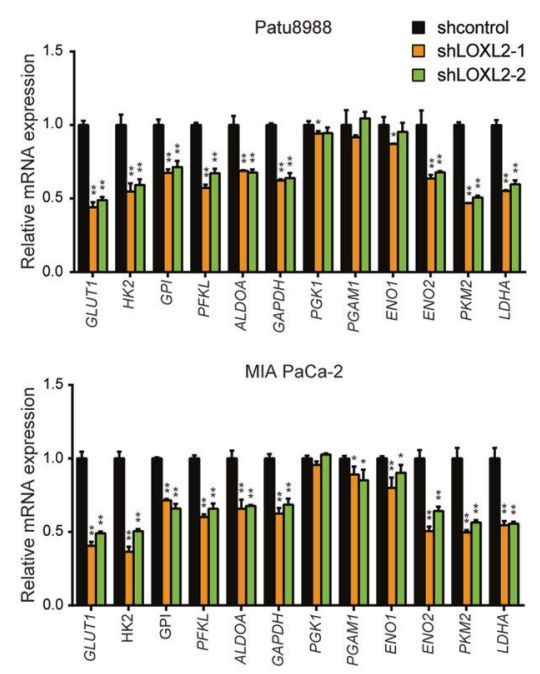

C

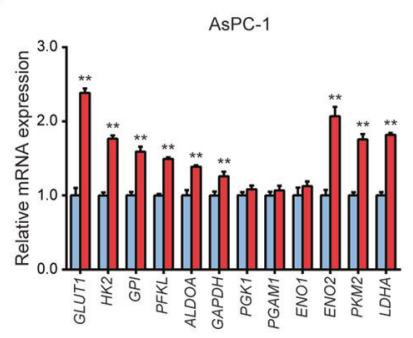

D

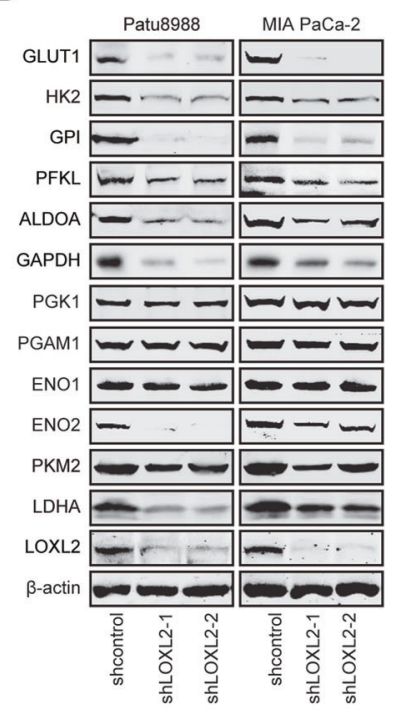

G
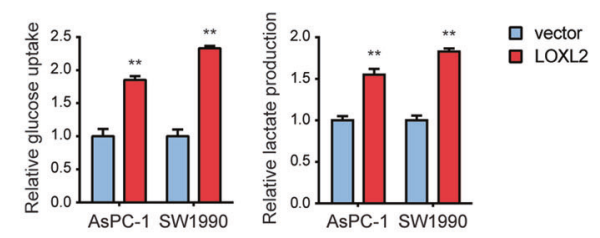

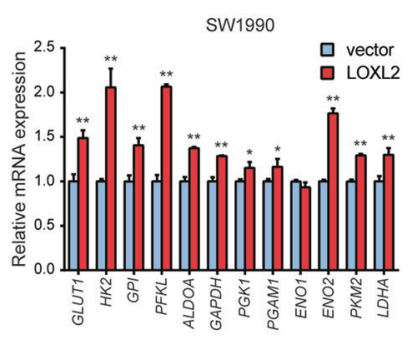

E

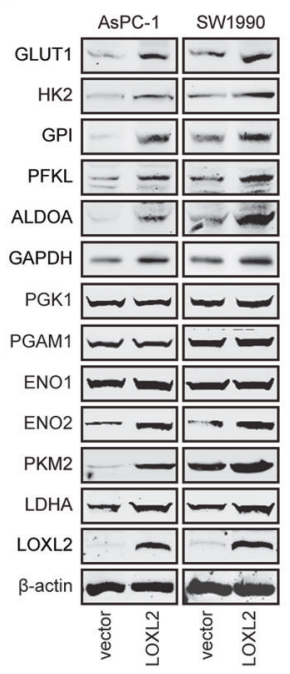

shcontrol

shLOXL2-2
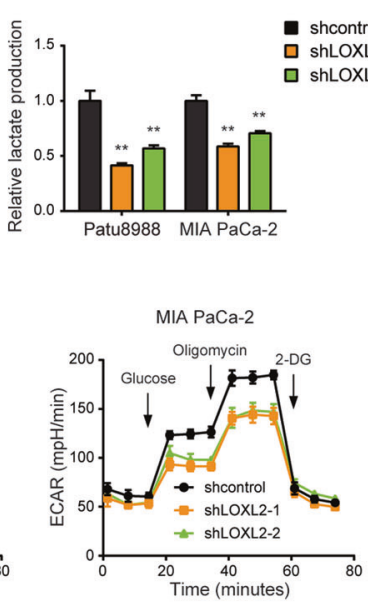

I

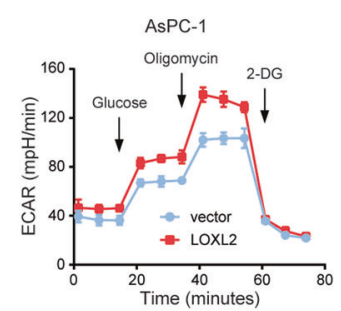

H

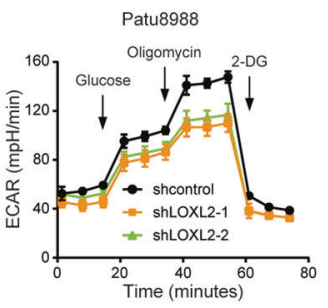

$\mathbf{F}$

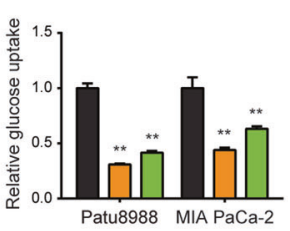

$\mathbf{J}$
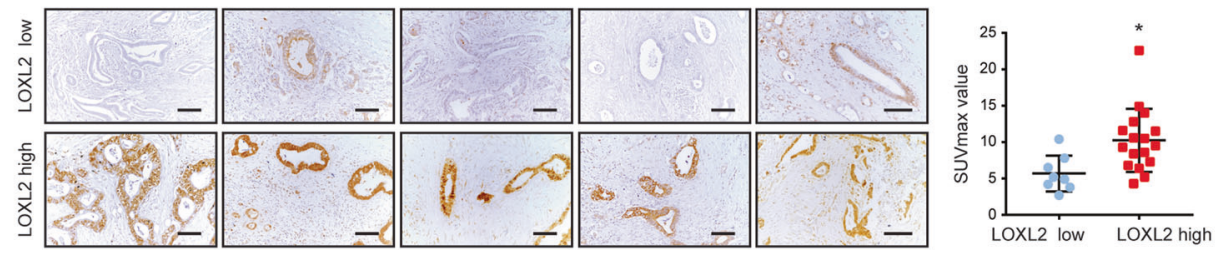

control cells, while both LOXL2 mutant cells had similar levels to vector control cells, confirming the absence of catalytic activity in LOXL2 mutants (Fig. 8C). Next, we tested whether or not LOXL2 depends on its catalytic activity to function as a positive regulator of PDAC. First, LOXL2 mutant cells had similar levels of HIF1a and hydroxylated HIF1a to control cells (Fig. 8D, E). Second, LOXL2 mutants failed to boost aerobic glycolysis (Fig. 8F) and the expression of glycolytic genes (Fig. S7). Third, LOXL2-mutant cells had similar proliferative, migratory, and invasive rates to those of control cells (Fig. 8G-I). Furthermore, we treated cells with the 
Fig. 5 LOXL2 enhances aerobic glycolysis in PDAC cells. A Positive correlation between LOXL2 expression and gene set HALLMARK GLYCOLYSIS in PDAC samples analyzed by GSEA based on the data from GEO and TCGA databases. NES Normalized enrichment score, FDR False discovery rates. B, C Analysis of mRNA levels of glycolytic genes in LOXL2 knockdown and control cells (B) as well as in LOXL2overexpressing and vector control cells (C) examined by qRT-PCR. D, E Analysis of protein levels of glycolytic genes in LOXL2 knockdown and control cells (D) as well as in LOXL2-overexpressing and vector control cells (E) examined by western blotting. F Glucose uptake and lactate production in LOXL2 knockdown and control cells. G Glucose uptake and lactate production in LOXL2-overexpressing and vector control cells. H Extracellular acid ratio (ECAR) in LOXL2 knockdown and control cells. I ECAR in LOXL2-overexpressing and vector control cells. J Representative IHC images of LOXL2 staining in PDAC tissues with the maximum standardized uptake value (SUVmax) (left panel) and comparison of the SUVmax between LOXL2-high and LOXL2-low patients (right panel). Scale bar: $50 \mu \mathrm{m} .{ }^{*} p<0.05,{ }^{* *} p<0.01$.

antioxidant $\mathrm{N}$-acetylcysteine (NAC) to suppress hydrogen peroxide and hence block the downstream effects of LOXL2 mediated potentially by intracellular hydrogen peroxide. Indeed, the higher HIF1a level in LOXL2-overexpressing cells could be attenuated by NAC treatment (Fig. 8J). Accordingly, NAC treatment hijacked the oncogenic activities induced by LOXL2 overexpression (Fig. S8A-D). Collectively, the catalytic activity of LOXL2 and its byproduct, hydrogen peroxide, is required for its oncogenic roles in PDAC.

\section{LOXL2 stimulates aerobic glycolysis and tumor progression via HIF1a}

To determine the involvement of HIF1a in LOXL2-mediated effects in PDAC, we depleted HIF1a in LOXL2-overexpressing cells (Fig. S9A, B). Depletion of HIF1a reversed enhanced aerobic glycolysis induced by LOXL2 overexpression (Fig. S9C). Moreover, depletion of HIF1a largely abolished the promoting effects of LOXL2 overexpression on cell proliferation, migration, and invasion (Fig. S9D-F). Together, these data indicate that HIF1a is indispensable for LOXL2-mediated tumor-promoting functions in PDAC cells.

\section{DISCUSSION}

LOXL2 has been considered as a tumor promoter, how precisely it contributes to malignant phenotypes remains incompletely understood. Our present study establishes a forward feedback loop between LOXL2 and HIF1a as a positive regulator of aerobic glycolysis, which is vital for PDAC progression (Fig. 9). The LOXL2 induction following hypoxia is like to potentiate HIF1a signaling via blocking HIF1a hydroxylation dependent on its catalytic activity through a hydrogen peroxide-mediated mechanism. Given that hypoxia is a common feature of tumor microenvironment that drives tumor progression and that metabolic reprogramming is considered a new target for cancer therapy [25-27], our study may help to better understand the oncogenic potential of LOXL2 in the hypoxic microenvironment and metabolic reprogramming.

LOXL2 overexpression is observed in many human cancers, including gastric cancer [28], hepatocellular carcinoma (HCC) [29], breast cancer [30], and squamous cell carcinomas [31], and closely associated with their clinical-pathological characteristics and prognosis. Importantly, LOXL2 functions as an oncogene in various human cancers via diverse mechanisms [32-34]. For instance, LOXL2 facilitates intrahepatic metastasis by crosslinking extracellular matrix components in the liver to promote cytoskeletal remodeling [29]; LOXL2 interacts and cooperates with Snail to downregulate E-cadherin expression, thus inducing epithelial-tomesenchymal transition (EMT) to drive carcinoma progression [33]. In pancreatic cancer, LOXL2 is involved in EMT induction, tumor progression, and gemcitabine resistance [35-37], but the underlying molecular mechanisms remain largely unknown. Consistent with LOXL2 promotion of tumor development and progression, LOXL2 knockdown and overexpression in human PDAC cell lines suggest oncogenic roles of LOXL2 in tumor growth and liver metastasis both in vitro and in vivo.
The Warburg effect is one of the most typical metabolic alterations in cancers. Emerging evidence supports the critical roles of the Warburg effect in promoting tumor initiation and progression [38]. Here, we demonstrated that LOXL2 could enhance aerobic glycolysis in PDAC cells, thus linking LOXL2 to tumor cell metabolic reprogramming. LOXL2 was found to upregulate the expression of multiple glycolytic genes, such as GLUT1, HK2, PKM2, and LDHA, leading to enhanced glycolytic activity. In addition, we showed that LOXL2-mediated glycolytic activation is required for its oncogenic roles in PDAC, suggesting a novel and critical role of LOXL2-mediated enhanced aerobic glycolysis in PDAC progression. With growing studies in tumor glucose metabolism, it has been realized that its increased activity is one of the major consequences of certain oncogenic drivers, among which HIF1a signaling represents the main pathway involved [39]. Intriguingly, LOXL2 indirectly enhances aerobic glycolysis in a HIF1a-dependent fashion. LOXL2 increases the levels of HIF1a protein and HIF1a depletion dramatically abrogates the promoting effects of LOXL2 overexpression on aerobic glycolysis as well as cell behaviors. Therefore, LOXL2mediated HIF1a regulation represents a previously unknown mechanism that links aerobic glycolysis to PDAC progression.

HIF1a is mainly regulated at the level of protein stability [40]. HIF1a is maintained at low levels under normoxic conditions by collaboration between hydroxylation controlled by PHD1-4 and ubiquitination controlled by the VHL-containing E3 ubiquitin ligase complex $[6,8]$. Here, LOXL2 was found to stabilize HIF1a via inhibiting PHD-dependent HIF1a hydroxylation upon the treatment of the PHD inhibitor DMOG. LOXL2 can exert its oncogenic roles via various mechanisms dependent or independent on its catalytic activity $[16,32,41-43]$. Hydrogen peroxide, as a byproduct of LOXL2 catalytic activity, belongs to ROS, which could inhibit the PHDs to stabilize HIF1a [22]. Our results indicated that the catalytic activity is indispensable for the oncogenic roles of LOXL2 in PDAC via generating LOXL2 mutants that lack the catalytic activity confirmed by measuring intracellular hydrogen peroxide levels. Moreover, LOXL2 functions as a PDCA promoter through a hydrogen peroxide-mediated mechanism. Taken together, LOXL2 enhances aerobic glycolysis via HIF1a stabilization dependent on its catalytic activity and thereby drives PDAC progression.

LOX family members have been reported to be induced by hypoxia and play critical roles in hypoxia-mediated tumor progression in several types of human cancers [44, 45]. In line with previous reports [46], LOXL2 expression in PDAC cells is induced by hypoxia in a HIF1a-dependent fashion. In view of the fact that LOXL2 stabilizes HIF1a, a feedforward loop is found between LOXL2 and HIF1a, which is confirmed by the observation in clinical PDAC tissues. A similar positive regulation loop between LOX and HIF1a was noticed in a previous study, which was proposed that the HIF1a-inducible LOX upregulates HIF1a protein synthesis via activating the PI3K-Akt signaling pathway dependent on LOX catalytic activity [46]. Distinct from this type of HIF1a regulation, our results uncover a HIF1a-regulatory mechanism in which LOXL2 upregulates HIF1a expression by stabilizing HIF1a 
A

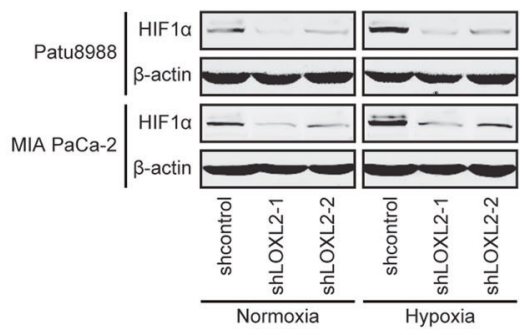

C

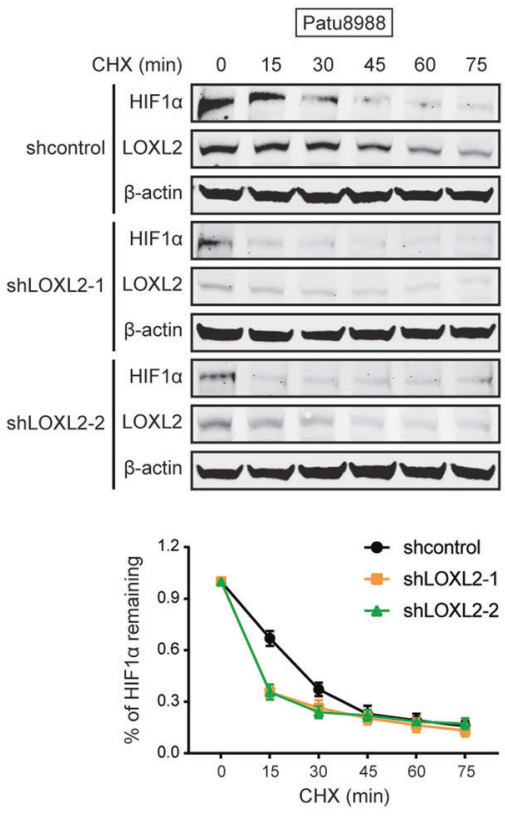

B

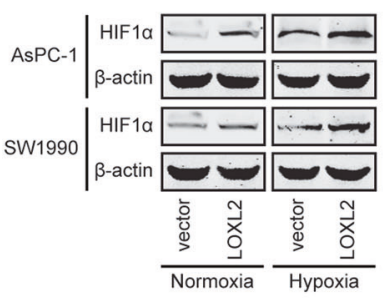

D

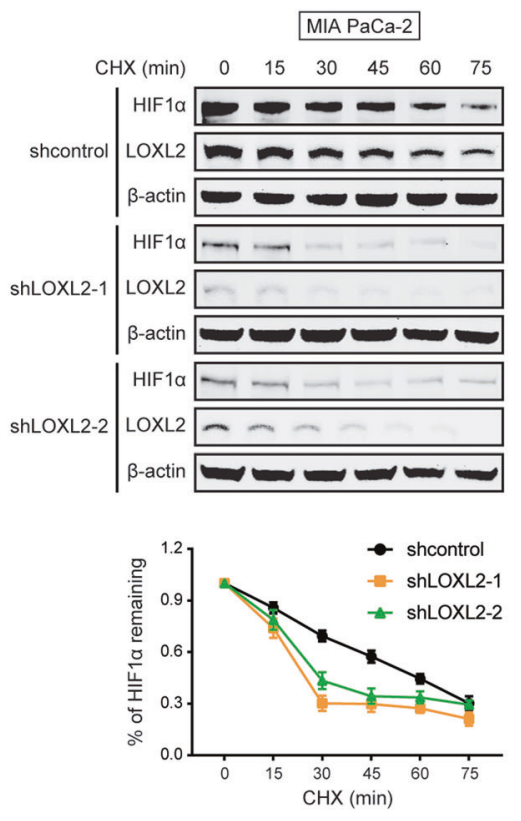

E
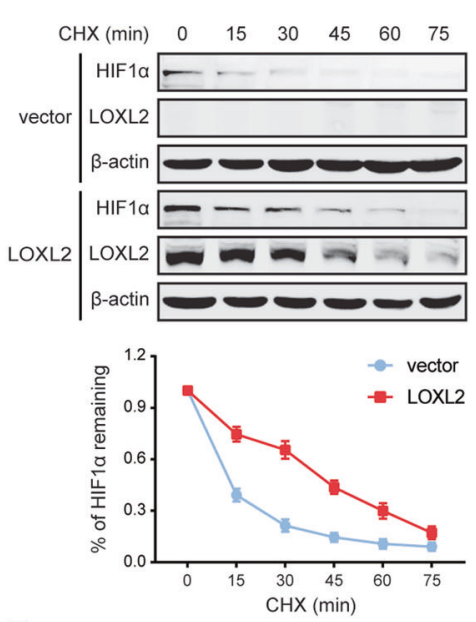

F

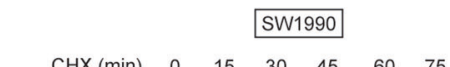

G

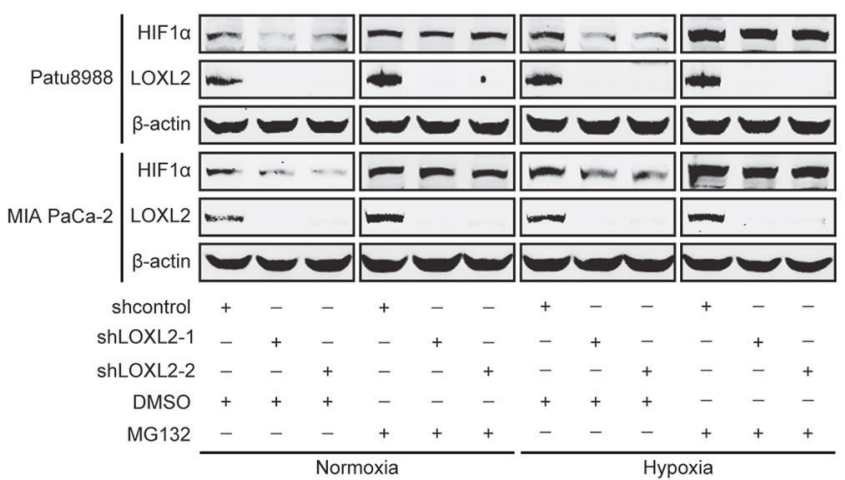

H

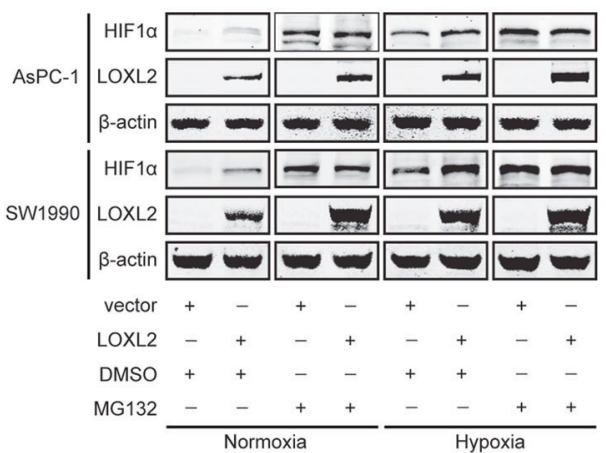

Fig. 6 LOXL2 stabilizes HIF1a protein. A Western blotting showing HIF1 $\alpha$ expression in LOXL2 knockdown and control cells under normoxic and hypoxic conditions. B Western blotting showing HIF1 $\alpha$ expression in LOXL2-overexpressing and vector control cells under normoxic and hypoxic conditions. C, D HIF1 $\alpha$ stability was examined by western blotting in LOXL2 knockdown and control cells. The cells were exposed to hypoxia for $6 \mathrm{~h}$ followed by incubation with $20 \mu \mathrm{g} / \mathrm{ml}$ cycloheximide (CHX) for the indicated times. E, F HIF1 $\alpha$ stability was examined by western blotting in LOXL2-overexprssing and vector control cells. The cells were exposed to hypoxia for $6 \mathrm{~h}$ followed by incubation with $20 \mu \mathrm{g} / \mathrm{ml} \mathrm{CHX}$ for the indicated times. G Western blotting showing HIF1 $\alpha$ expression in LOXL2 knockdown and control cells treated with or without $10 \mu \mathrm{M} \mathrm{MG} 132$ for $6 \mathrm{~h}$ under normoxic and hypoxic conditions. H Western blotting showing HIF1 $\alpha$ expression in LOXL2-overexpressing and vector control cells treated with or without $10 \mu \mathrm{M} \mathrm{MG132}$ for $6 \mathrm{~h}$ under normoxic and hypoxic conditions.

from PHD-mediated hydroxylation, providing new molecular insights into how HIF1a is regulated by the LOX family.

LOXL2 has a complex role in cancers, which could be dependent or independent on its enzymatic activity, and at the same time, on its intracellular, extracellular, or intranuclear forms $[47,48]$. Here, we propose the LOXL2-mediated stabilization of HIF1a in regulating PDAC progression and aerobic glycolysis dependent on its enzymatic activity through a hydrogen peroxide- 
A

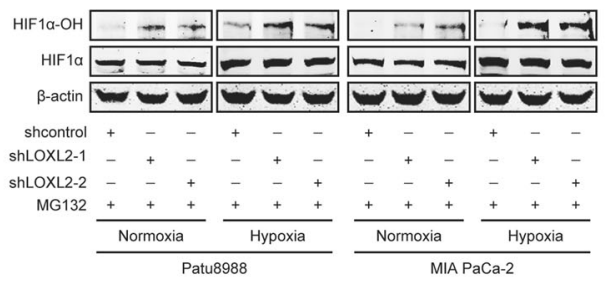

C

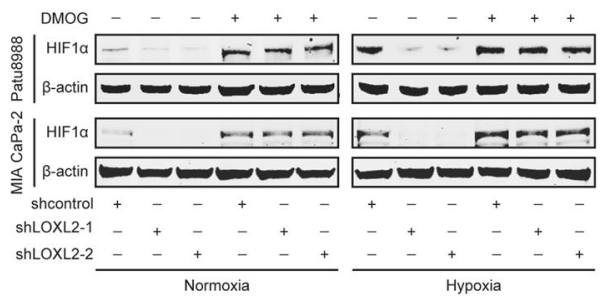

B

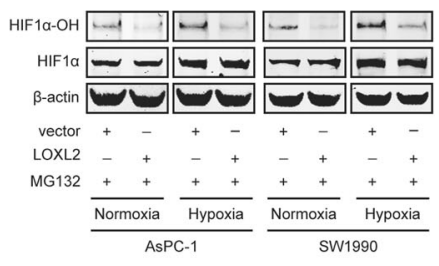

D

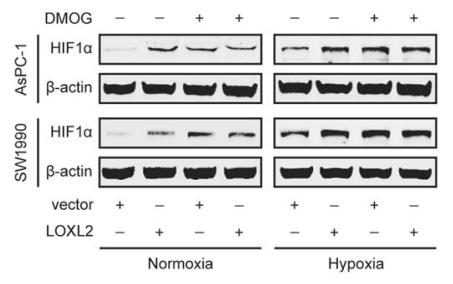

Fig. 7 LOXL2 inhibits prolyl hydroxylase (PHD)-dependent hydroxylation of HIF1a. A Western blotting showing amounts of hydroxylated HIF1 $\alpha(\mathrm{HIF} 1 \alpha-\mathrm{OH})$ and HIF1 $\alpha$ in LOXL2 knockdown and control cells treated with $10 \mu \mathrm{M} \mathrm{MG132}$ for $6 \mathrm{~h}$ under normoxic and hypoxic conditions. B Western blotting showing amounts of HIF1 $\alpha-\mathrm{OH}$ and HIF1 $\alpha$ in LOXL2-overexpressing and vector control cells treated with $10 \mu \mathrm{M}$ MG132 for $6 \mathrm{~h}$ under normoxic and hypoxic conditions. C Western blotting showing HIF1 $\alpha$ expression in LOXL2 knockdown and control cells treated with or without $1 \mathrm{mM}$ dimethyloxalylglycine (DMOG) for $24 \mathrm{~h}$ under normoxic and hypoxic conditions. D Western blotting showing HIF1 $\alpha$ expression in LOXL2-overexpressing and vector control cells treated with or without $1 \mathrm{mM}$ DMOG for $24 \mathrm{~h}$ under normoxic and hypoxic conditions.

mediated mechanism, but the involvement of other molecular mechanisms should not be ruled out, as LOXL2 is multi-functional. LOXL2 plays crucial intranuclear roles in the regulation of chromatin structure and gene transcription by mediating the oxidative deamination of lysine residues on target proteins, such as Snail1, H3K4me3, and TAF10 [49-51]. LOXL2 interacts with Snail1 to increase Snail1 stability [52], or to regulate heterochromatin transcription [49], thus inducing EMT. Unlike Snial1, despite intensive LOXL2 expression observed in the nuclei of PDAC tissues and cells, we did not detect the interaction between LOXL2 and HIF1a. Nevertheless, intranuclear roles of LOXL2 in PDAC warrant further study.

\section{CONCLUSIONS}

Our study uncovers a previously unprecedented function of LOXL2 in PDAC progression via promoting aerobic glycolysis. Moreover, our results provide new mechanistic insights into the crucial roles of LOXL2 in HIF1a stabilization via inhibiting its hydroxylation dependent on its catalytic byproduct, hydrogen peroxide. The present suggests the feasibility of targeting the LOXL2-HIF1a feedback loop to inhibit aerobic glycolysis and to reverse the malignancy of PDAC, a cancer type for which existing therapeutic options are clinically insufficient.

\section{MATERIALS AND METHODS \\ Clinical samples and IHC staining}

Paraffin-embedded PDAC specimens were histopathologically diagnosed by the Department of Pathology, Ren Ji Hospital, School of Medicine, Shanghai Jiao Tong University. Two cohorts of specimens were used in this study: a tissue microarray containing 205 pathologist-certified and clinically annotated PDAC specimens and corresponding non-cancerous tissues and 25 PDAC tissues with available information of preoperative ${ }^{18} \mathrm{~F}$-FDG PET/CT. All the patients did not receive chemotherapy, radiotherapy, or other adjuvant therapies prior to the surgery. Written informed consent was obtained from each patient. The use of these tissue samples was approved by the ethical review committee of the World Health Organization Collaborating Center for Research in Human Production (authorized by the Shanghai Municipal Government). IHC staining was performed using a two-step protocol as previously described [53]. For IHC analysis on xenograft tumors and pancreas tissues from KPC mice established by our laboratory [38], the specimens were fixed in $4 \%$ paraformaldehyde, embedded in paraffin and subjected to $\mathrm{IHC}$ staining. Primary antibodies used for IHC staining were LOXL2 (1:500, GeneTex, GTX105085) and PCNA (1:300, Cell Signaling Technology, \#13110). Scoring was conducted based on both the ratio and intensity of the staining as previously reported [54].

\section{Cell culture and reagents}

The human PDAC cell lines used in this study, including AsPC-1, BxPC-3, Capan-1, CFPAC-1, HPAC, MIA PaCa-2, PANC-1, Patu8988, and SW1990 were all preserved in Shanghai Cancer Institute, Ren Ji Hospital, School of Medicine, Shanghai Jiao Tong University. All PDAC cell lines were cultured in suggested medium (Hyclone, Logan, UT, USA) according to ATCC protocols, supplemented with $10 \%(\mathrm{v} / \mathrm{v})$ fetal bovine serum (FBS, Hyclone, USA) and 100 Units $/ \mathrm{mL}$ penicillin and $100 \mu \mathrm{g} / \mathrm{mL}$ streptomycin (Invitrogen, USA) at $37{ }^{\circ} \mathrm{C}$ in a humidified incubator with $5 \% \mathrm{CO}_{2}$, except for MIA PaCa2 , which was cultured in medium supplemented with $10 \%(\mathrm{v} / \mathrm{v}) \mathrm{FBS}, 2.5 \%$ $(\mathrm{v} / \mathrm{v})$ horse serum (Hyclone, USA), and $100 \mathrm{Units} / \mathrm{mL}$ penicillin and $100 \mu \mathrm{g} /$ $\mathrm{mL}$ streptomycin. For hypoxic culture, PDAC cells were grown in a hypoxia incubator in an atmosphere consisting of $1 \% \mathrm{O}_{2}, 94 \% \mathrm{~N}_{2}$, and $5 \% \mathrm{CO}_{2}$. The reagents used in the study were listed as follows: 2-DG (Sigma-Aldrich, D8375), CHX (Sigma-Aldrich, 239763-M), DMOG (Selleck, S7483), galactose (Sigma-Aldrich, G5388), MG132 (Selleck, S2619), and NAC (Sigma-Aldrich, A7250).

\section{Western blotting and co-immunoprecipitation}

Standard western blotting was carried out using whole-cell protein lysates. Briefly, cultured cells were lysed with IP lysis buffer (Beyotime, Shanghai, China) supplemented with protease and phosphatase inhibitor cocktail (Bimake.cn, Houston, TX). Protein concentrations were determined using the Pierce BCA Protein Assay Kit (Thermo Fisher Scientific, USA) according to the manufacturer's instructions. Cell lysates were analyzed by $5-10 \%$ sodium dodecyl sulfate-polyacrylamide gel electrophoresis (SDS-PAGE). Nitrocellulose (NC) membrane (Millipore, Danvers, MA) was used for gel transfer. After blocking with $5 \%$ non-fat milk, the membranes were hybridized overnight with primary antibodies: LOXL2 (1:1000, GeneTex, GTX105085), HIF1a (1:1000, Abcam, ab2185), Hydroxy HIF1a (1:1000, Cell Signaling Technology, \#3434), GLUT1 (1:1000, Proteintech, 21829-1-AP), HK2 (1:5000, Proteintech, 22029-1-AP), GPI (1:1000, Proteintech, 15171-1AP), PFKL (1:200, Santa Cruz, sc-393713), ALDOA (1:10000, Proteintech, 11217-1-AP), GAPDH (1:50000, Proteintech, 60004-1-lg), PGK1 (1:1200, Proteintech, 17811-1-AP), PGAM1 (1:1000, Proteintech, 16126-1-AP), ENO1 
A

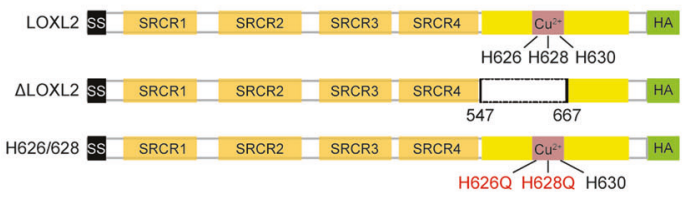

B

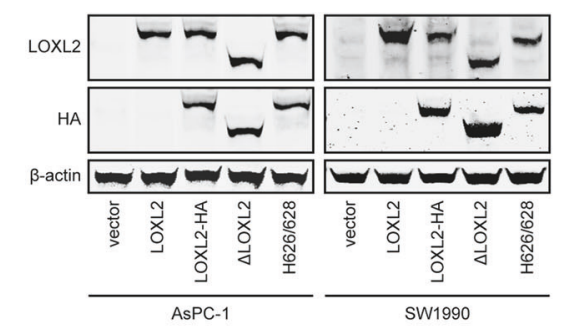

D

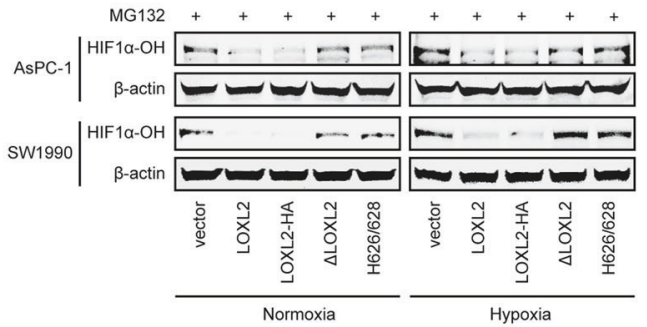

$\mathbf{F}$
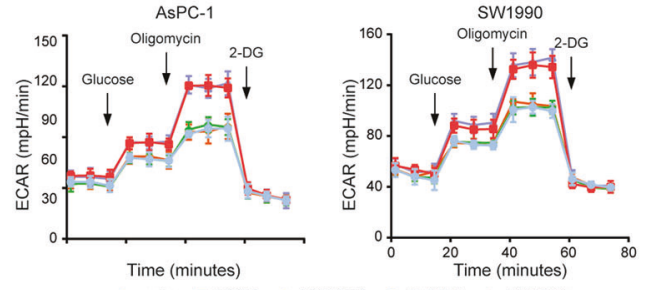

$\rightarrow$ vector $\Rightarrow$ LOXL2 $\$$ LOXL2-HA $\rightarrow \Delta$ LOXL2 $\rightarrow$ H626/628

$\mathbf{J}$

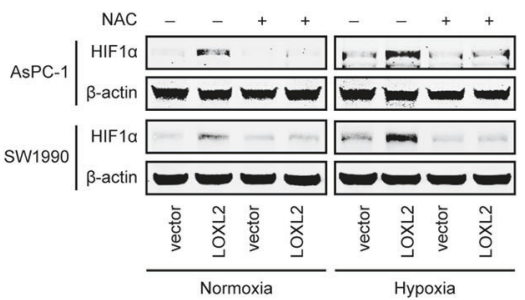

H
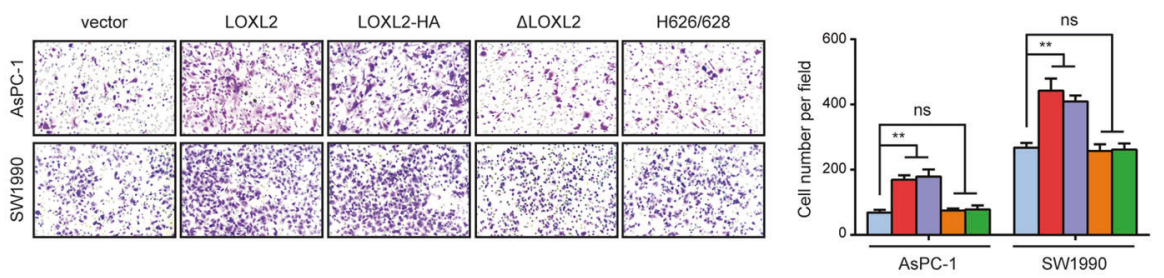

$\square$ vector

$\square$ LOXL2-HA

$\square \mathrm{LOXL2}$

$H 626 / 628$

I
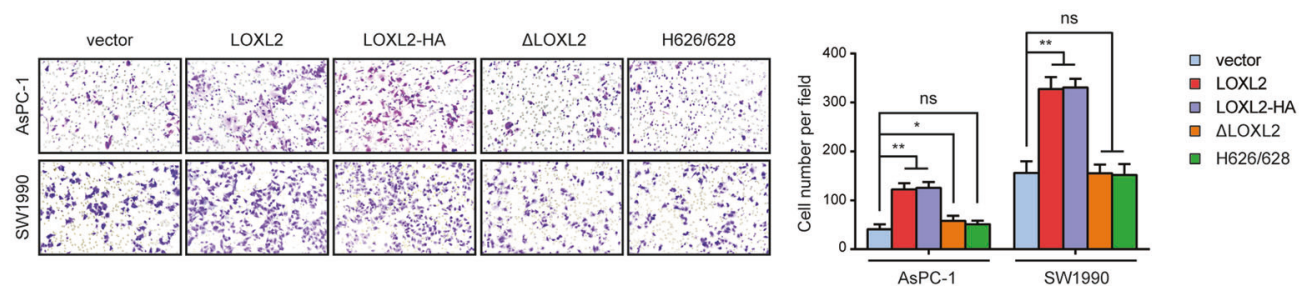

(1:1000, Proteintech, 11204-1-AP), ENO2 (1:1000, Protrintech, 66150-1-lg), PKM2 (1:1000, Cell Signaling Technology, \#3198), LDHA (1:5000, Proteintech, 19987-1-AP), HA (1:1000, Millipore, 05-904), and $\beta$-actin (1:5000, MultiSciences, ab008). The next day, secondary antibodies conjugated with DyLight fluorescent dye (Thermo Fisher Scientific, USA) were incubated and the signal was detected using an Infrared Odyssey Imaging System (LI-
COR Biosciences, Lincoln, NE, USA). For co-immunoprecipitation, cells were lysed as described in western blotting for $15 \mathrm{~min}$ on ice and supernatant was collected after centrifuging at $12,000 \mathrm{~g}$ for $15 \mathrm{~min}$ at $4{ }^{\circ} \mathrm{C}$. Cell lysates were incubated with Pierce Anti-HA Magnetic Beads (Thermo Fisher Scientific, USA, \#88836), anti-HIF1a ( $2 \mu \mathrm{g}$, Abcam, ab1), and anti-IgG (as a negative control, $2 \mu \mathrm{g}, \mathrm{Abcam}$, ab200699) with rotation for $30 \mathrm{~min}$ at room 
Fig. 8 The catalytic activity is responsible for the oncogenic roles of LOXL2. A Schematic representation of wild-type LOXL2, deletion mutant $(\Delta \mathrm{LOXL2})$, and double point mutant $(\mathrm{H} 626 \mathrm{Q} / \mathrm{H} 628 \mathrm{Q})$ with the HA-tag. B Western blotting showing the indicated LOXL2 variants stably expressed in PDAC cells using anti-LOXL2 and anti-HA antibodies. C Intracellular hydrogen peroxide measured in PDAC cells stably transfected with the indicated LOXL2 variants. D Western blotting showing hydroxylated HIF1 $\alpha$ (HIF $1 \alpha-\mathrm{OH})$ expression in PDAC cells stably transfected with the indicated LOXL2 variants treated with $10 \mu \mathrm{M}$ MG132 for $6 \mathrm{~h}$ under normoxic and hypoxic conditions. E Western blotting showing HIF1 $\alpha$ expression in PDAC cells stably transfected with the indicated LOXL2 variants under normoxic and hypoxic conditions. $\mathbf{F}$ Extracellular acid ratio (ECAR) in PDAC cells stably transfected with the indicated LOXL2 variants. G CCK-8 assay of PDAC cells stably transfected with the indicated LOXL2 variants. $\mathbf{H}$, I Transwell migration $(\mathbf{H})$ and invasion (I) assays of PDAC cells stably transfected with the indicated LOXL2 variants. J Western blotting showing HIF1 $\alpha$ expression in LOXL2-overexpressing and vector control cells treated with $10 \mathrm{mM} \mathrm{N}$-acetylcysteine (NAC) for $24 \mathrm{~h}$ under normoxic and hypoxic conditions. ${ }^{*} p<0.05,{ }^{* *} p<0.01$, ns No significance.

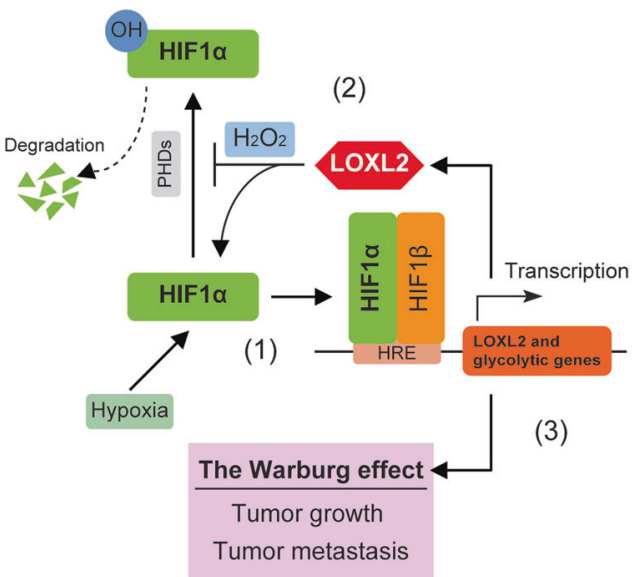

Fig. 9 Model illustrating the mechanism regulated by LOXL2 in PDAC progression. 1 Hypoxia induces LOXL2 expression in a HIF1 $\alpha$ dependent fashion. 2 LOXL2 inhibits the hydroxylation of HIF1 $\alpha$ to stabilize it via hydrogen peroxide $\left(\mathrm{H}_{2} \mathrm{O}_{2}\right)$, the byproduct of LOXL2 catalytic activity, resulting in the expression of multiple glycolytic genes. 3 LOXL2 drives pancreatic cancer growth and metastatic progression dependent on the Warburg effect.

temperature (RT), followed by incubation with Pierce Protein-A/G Magnetic Beads (Thermo Fisher Scientific, USA, \#88803) for 15 min at RT. Immunocomplexes were washed three times with TBS-T or PBS-T and then resuspended in $1 \times$ SDS-PAGE sample buffer for western blotting analysis.

\section{Quantitative real-time polymerase chain reaction (qRT-PCR) \\ Total RNA was extracted from cultured cells using the TRIzol total RNA isolation reagent (Takara Bio, Japan). Complementary DNA (cDNA) was prepared from $500 \mathrm{ng}$ of total RNA using a prime Script RT reagent kit (Takara Bio, Japan) and subsequently subjected to qRT-PCR on ABI7500 instrument (Applied Biosystems) using $2 \times$ SYBR Green qPCR Master Mix (Bimake, Shanghai, China) and specific primers listed in the Table S2. The expression of target genes was normalized to human $18 s R N A$.}

\section{Immunofluorescence analysis}

All the PDAC cells used in this study were seeded on the confocal dishes and incubated overnight. Cells were fixed in $4 \%$ paraformaldehyde for $30 \mathrm{~min}$, permeabilized with $0.2 \%$ Triton $\mathrm{X}-100$ for $2.5 \mathrm{~min}$, blocked in $1 \%$ bovine serum albumin (BSA) for $60 \mathrm{~min}$, and incubated with primary antibodies against LOXL2 (1:200, GeneTex, GTX105085) for $60 \mathrm{~min}$ at RT, followed by Alexa Fluor 594-conjugated secondary antibodies (Molecular Probes, USA) for $30 \mathrm{~min}$ at RT. Nuclei were counterstained with 4',6diamidino-2-phenylindole (DAPI) (Vector Laboratories, Burlingame, CA, USA). Immunofluorescence signals were captured using laser confocal microscopy (Leica Microsystems AG).

\section{Transfection with siRNA}

Small interfering RNAs (siRNAs) targeting HIF1a (siHIF1a) and nontargeting siRNAs (siNC) were synthesized by GenePharma Inc. (Shanghai, China) and transfected into PDAC cells according to the manufacturer's instruction. Briefly, PDAC cells at 40 to $60 \%$ confluence were transfected with siRNAs using Lipofectamine 2000 (Invitrogen, Carlsbad, CA, USA) in
optiMEM medium (Gibco, Invitrogen, Carlsbad, Calif, USA) for $6 \mathrm{~h}$ and then cultured in complete medium for another $48 \mathrm{~h}$, followed by efficiency validation and subsequent function assays. The sequences targeting HIFla gene were listed as follows: siHIF1a-1 (HIF1a-Homo-1168, 5'-3': GAUGAAA GAAUUACCGAAUTT, 5'-3' AUUCGGUAAUUCUUUCAUCTT), siHIF1a-2 (HIF1 a-Homo-2090, 5'-3': CUCCCUAUAUCCCAAUGGATT, 5'-3' UCCAUUGGGAUA UAGGGAGTT).

\section{Lentivirus production and transfection}

For overexpression, the plasmids expressing LOXL2, LOXL2-HA, and LOXL2 mutants were constructed by Shanghai Generay Biotech Co., Ltd. The cDNAs encoding full-length human LOXL2 (NM_002318), LOXL2-HA, and LOXL2 mutants were synthesized and inserted into PCDH-CMV-MCS-EF1Puro vector (System Biosciences, Palo Alto, CA, USA). For knockdown, short hairpin RNA (shRNA) plasmids targeting LOXL2 were purchased from Shanghai Genechem Co., LTD. shRNA sequences targeting LOXL2 gene were listed as follows: shLOXL2-1: 5'-GAAGGAGACATCCAGAAGAAT-3'; shLOXL2-2: 5'-GAGAGGACATACAATACCAAA-3'. 293T packaging cells were used to produce lentivirus, which was then transfected into target cell lines with $6 \mu \mathrm{g} / \mathrm{ml}$ polybrene for $24 \mathrm{~h}$. Transfected cells used for overexpression or knockdown and their control cells were selected with $5 \mu \mathrm{g} / \mathrm{ml}$ puromycin for 2 weeks. The overexpression or knockdown efficiency of LOXL2 was assessed by qRT-PCR and western blotting.

\section{Hydrogen peroxide measurement}

Intracellular hydrogen peroxide was measured using the Amplex Red Hydrogen Peroxide/Peroxidase Assay Kit (Invitrogen, Carlsbad, CA, USA) by a spectrophotometer according to the manufacturer's instruction.

\section{Cell viability assay}

Cell viability was determined using a Cell Counting Kit (CCK-8, Dojindo, Japan) according to the manufacturers' instructions. Briefly, cells $(1-2 \times$ $10^{3}$ cells per well) were seeded into 96-well plates. The culture medium was removed and $100 \mu \mathrm{l}$ of a 1:10 (v/v) dilution of CCK-8 in the medium was added to each well at the indicated time points. After incubated for $30-90 \mathrm{~min}$ in the cell incubator, the plates were measured at $450 \mathrm{~nm}$ using a multifunctional microplate reader (Bio-Rad Laboratories, Hercules, CA).

\section{Colony formation assay}

Cells $\left(1 \times 10^{3}\right.$ cells per plate) were seeded in $6 \mathrm{~cm}$ plates and allowed to grow for approximately 14 days. At the end of the experiments, colonies formed were washed with PBS twice, fixed with $4 \%$ paraformaldehyde for $30 \mathrm{~min}$, and stained with $0.2 \%$ crystal violet for $1 \mathrm{~h}$. Colonies larger than $100 \mu \mathrm{m}$ in diameter for each plate were counted.

\section{Transwell migration and invasion assays}

The capacity of cell migration and invasion was measured using $8-\mu \mathrm{m}$-pore Transwell chambers (Millipore, USA). For migration assay, $5 \times 10^{4}$ cells in $200 \mu \mathrm{l} \mathrm{serum}$-free medium were seeded into the upper chamber and allowed to migrate for $24 \mathrm{~h}$. For invasion assay, $1 \times 10^{5}$ cells in $200 \mu \mathrm{l}$ serum-free medium were seeded into the upper chamber coated with Matrigel (BD Biosciences) and allowed to invade for $48 \mathrm{~h}$. Meanwhile, $750 \mu \mathrm{l}$ medium with $10 \% \mathrm{FBS}$ in the lower chamber acted as a chemoattractant. 2-DG, galactose, and NAC were also added into the lower chamber. Subsequently, cells were fixed with $4 \%$ paraformaldehyde for $30 \mathrm{~min}$ and stained with $0.2 \%$ crystal violet for $1 \mathrm{~h}$, followed by removing non-invading cells on the top surface of the chamber with cotton swabs. Finally, stained cells were counted in five randomly selected 
fields with $200 \times$ magnification under a microscope to minimize the bias.

\section{Glucose consumption and lactate production assays}

Cells were cultured to about $40 \%$ confluency in 6 -well plates, and then incubated in fresh culture medium for additional $24 \mathrm{~h}$. The culture medium was collected by centrifugation to remove the cells, and the levels of glucose (Catalog \#: K676) and lactate (Catalog \#: K607) were determined using kits from BioVision according to the manufacturer's instructions. The values were normalized to the total protein amount determined using the Pierce BCA Protein Assay Kit (Thermo Fisher Scientific).

\section{Extracellular acidification rate (ECAR) and oxygen consumption rate (OCR) assays}

The Seahorse XF96 Flux Analyzer (Seahorse Bioscience, Billerica, Massachusetts, USA) was used to measure ECAR and OCR of PDAC cells according to the manufacturer's instructions. Approximately $1-2 \times 10^{5}$ cells per well were seeded into an XF96-well plate and attached overnight. For the assessment of ECAR, cells were incubated with non-buffered RPMI 1640 under basal conditions followed by sequential injection of $10 \mathrm{mM}$ glucose, $1 \mathrm{mM}$ mitochondrial poison (oligomycin, Sigma-Aldrich, Saint Louis, Missouri, USA) and $80 \mathrm{mM}$ glycolysis inhibitor (2-deoxyglucose, 2-DG, Sigma-Aldrich). OCR was assessed under basal conditions and after a sequential injection of $1 \mu \mathrm{M}$ oligomycin, $1 \mu \mathrm{M}$ fluoro-carbonyl cyanide phenylhydrazone (FCCP, SigmaAldrich, Saint Louis, Missouri, USA) and $2 \mathrm{mM}$ antimycin $\mathrm{A}$ and rotenone (Sigma-Aldrich, Saint Louis, Missouri, USA). Both ECAR and OCR measurements were normalized by total protein content.

\section{Measurement of the half-life of HIF1a protein}

Cells were seeded in $6-\mathrm{cm}$ plates and exposed to hypoxia for $6 \mathrm{~h}$ to induce HIF1a protein. Then, $\mathrm{CHX}$, a protein synthesis inhibitor, was used to treat the cells at a concentration of $20 \mu \mathrm{g} / \mathrm{ml}$ for $15,30,45,60$, and $75 \mathrm{~min}$ as indicated. The cell lysis was harvested followed by western blotting. The scale of the bands was analyzed by ImageJ software.

Subcutaneous xenograft and intrasplenic inoculation models In the subcutaneous model, $2 \times 10^{6}$ cells (LOXL2-overexpressing AsPC-1 cells, LOXL2 knockdown Patu8988 cells, and their control cells) resuspended in $100 \mu \mathrm{l} \mathrm{PBS}$ were injected subcutaneously into the back of Balb/c nude mice (male, 5-6-week old, five mice per group). 5 weeks after subcutaneous inoculation, tumors were resected, weighed, embedded in paraffin, and subjected to IHC staining. In the intrasplenic inoculation model, $2 \times 10^{6}$ cells (LOXL2-overexpressing AsPC-1 cells, LOXL2 knockdown Patu8988 cells, and their control cells) resuspended in $25 \mu \mathrm{l}$ PBS were injected into the spleen of Balb/c nude mice (male, 5-6-week old, five mice per group). Mice harboring LOXL2 knockdown Patu8988 cells and their control cells were sacrificed 5 weeks after implantation, while mice harboring LOXL2-overexpressing AsPC-1 cells and their vector control cells were sacrificed 24 days after implantation, when mice harboring LOXL2-overexpressing AsPC-1 cells appeared with cachexia. The livers were dissected, fixed with 4\% paraformaldehyde, embedded in paraffin, and subjected to hematoxylin and eosin (H\&E) staining. Mice were manipulated and housed according to protocols approved by the East China Normal University Animal Care Commission. All mice received humane care according to the criteria outlined in the Guide for the Care and Use of Laboratory Animals prepared by the National Academy of Sciences and published by the National Institutes of Health.

\section{Bioinformatics analysis}

The Gene Expression Omnibus (GEO) data and The Cancer Genome Atlas (TCGA) data referenced in the study are available in a public repository from the GEO website (https://www.ncbi.nlm.nih.gov/geo/) and the TCGA website (https://cancergenome.nih.gov/). LOXL2 gene expression analysis was conducted using microarray gene expression data sets with the accession codes GSE15471, GSE16515, GSE28735, GSE32676, GSE60980, GSE62165, GSE62452, GSE71729, and GSE102238. Gene set enrichment analysis (GSEA, http://www.broadinstitute.org/gsea/index.jsp) was carried out based on the data from GEO and TCGA data sets. The pancreatic cancer samples in each data set were divided into two groups by the mean LOXL2 expression level. GSEA was performed to compare the two groups within the Molecular Signatures Database of (h.all.v6.2.symbols.gmt). The gene sets showing a nominal $p$ value less than 0.05 and false discovery rates (FDR) less than 0.25 were considered significantly enriched.
Statistical analysis

Statistical analyses were performed using SPSS 19.0 for windows (IBM Corporation) and GraphPad Prism 7 software (San Diego, CA). The results were shown as mean $\pm S D$ and compared using a two-tailed, unpaired Student's t-test or analysis of variance (ANOVA). Significant differences were accepted for $p$ values of $<0.05$.

\section{DATA AVAILABILITY}

All data needed to evaluate the conclusions in the paper are present in the paper and/or the Supplementary Materials. Additional data related to this paper may be requested from the authors.

\section{REFERENCES}

1. Ryan DP, Hong TS, Bardeesy N. Pancreatic adenocarcinoma. N. Engl J Med. 2014;371:1039-49.

2. Siegel RL, Miller KD, Jemal A. Cancer statistics, 2018. CA Cancer J Clin. 2018;68:7-30.

3. Rahib L, Smith BD, Aizenberg R, Rosenzweig AB, Fleshman JM, Matrisian LM. Projecting cancer incidence and deaths to 2030: the unexpected burden of thyroid, liver, and pancreas cancers in the United States. Cancer Res. 2014;74:2913-21.

4. Neesse A, Bauer CA, Ohlund D, Lauth $M$, Buchholz M, Michl P, et al. Stromal biology and therapy in pancreatic cancer: ready for clinical translation? Gut. 2019;68:159-71.

5. Ruan K, Song G, Ouyang G. Role of hypoxia in the hallmarks of human cancer. J Cell Biochem. 2009;107:1053-62.

6. Ivan $\mathrm{M}$, Kondo $\mathrm{K}$, Yang $\mathrm{H}$, Kim W, Valiando J, Ohh $\mathrm{M}$, et al. HIFalpha targeted for VHL-mediated destruction by proline hydroxylation: implications for $\mathrm{O} 2$ sensing. Science. 2001;292:464-8.

7. Keith $B$, Johnson RS, Simon MC. HIF1alpha and HIF2alpha: sibling rivalry in hypoxic tumour growth and progression. Nat Rev Cancer. 2011;12:9-22.

8. Jaakkola P, Mole DR, Tian YM, Wilson MI, Gielbert J, Gaskell SJ, et al. Targeting of HIF-alpha to the von Hippel-Lindau ubiquitylation complex by O2-regulated prolyl hydroxylation. Science. 2001;292:468-72.

9. Cairns RA, Harris IS, Mak TW. Regulation of cancer cell metabolism. Nat Rev Cancer. 2011;11:85-95.

10. Liberti MV, Locasale JW. The warburg effect: how does it benefit cancer cells? Trends Biochem Sci. 2016;41:211-8.

11. Menendez JA, Joven J, Cufi S, Corominas-Faja B, Oliveras-Ferraros C, Cuyas E, et al. The Warburg effect version 2.0: metabolic reprogramming of cancer stem cells. Cell Cycle. 2013;12:1166-79.

12. Doherty JR, Cleveland JL. Targeting lactate metabolism for cancer therapeutics. J Clin Invest. 2013;123:3685-92.

13. de la Cruz-Lopez KG, Castro-Munoz LJ, Reyes-Hernandez DO, Garcia-Carranca A Manzo-Merino J. Lactate in the regulation of tumor microenvironment and therapeutic approaches. Front Oncol. 2019;9:1143.

14. Chen W, Yang A, Jia J, Popov YV, Schuppan D, You H. Lysyl Oxidase (LOX) family members: rationale and their potential as therapeutic targets for liver fibrosis. Hepatology. 2020;72:729-41.

15. Vallet SD, Ricard-Blum S. Lysyl oxidases: from enzyme activity to extracellular matrix cross-links. Essays Biochem. 2019;63:349-64.

16. Wen B, Xu LY, Li EM. LOXL2 in cancer: regulation, downstream effectors and novel roles. Biochim Biophys Acta Rev Cancer. 2020;1874:188435.

17. Ye Y, Hu Q, Chen H, Liang K, Yuan Y, Xiang Y, et al. Characterization of hypoxiaassociated molecular features to aid hypoxia-targeted therapy. Nat Metab. 2019;1:431-44.

18. Semenza GL. HIF-1 mediates metabolic responses to intratumoral hypoxia and oncogenic mutations. J Clin Invest. 2013;123:3664-71.

19. Lee JY, Park JH, Choi HJ, Won HY, Joo HS, Shin DH, et al. LSD1 demethylates HIF1alpha to inhibit hydroxylation and ubiquitin-mediated degradation in tumor angiogenesis. Oncogene. 2017;36:5512-21.

20. Oh ET, Kim JW, Kim JM, Kim SJ, Lee JS, Hong SS, et al. NQO1 inhibits proteasomemediated degradation of HIF-1alpha. Nat Commun. 2016;7:13593.

21. Lee HY, Lee $T$, Lee $N$, Yang EG, Lee $C$, Lee J, et al. Src activates HIF-1 alpha not through direct phosphorylation of HIF-1alpha specific prolyl-4 hydroxylase 2 but through activation of the NADPH oxidase/Rac pathway. Carcinogenesis. 2011;32:703-12.

22. Galanis A, Pappa A, Giannakakis A, Lanitis E, Dangaj D, Sandaltzopoulos R. Reactive oxygen species and HIF-1 signalling in cancer. Cancer Lett. 2008;266:12-20.

23. Cuevas EP, Moreno-Bueno G, Canesin G, Santos V, Portillo F, Cano A. LOXL2 catalytically inactive mutants mediate epithelial-to-mesenchymal transition. Biol Open. 2014;3:129-37.

24. Herranz N, Dave N, Millanes-Romero A, Pascual-Reguant L, Morey L, Diaz VM, et al. Lysyl oxidase-like 2 (LOXL2) oxidizes trimethylated lysine 4 in histone H3. FEBS J. 2016;283:4263-73. 
25. Hay N. Reprogramming glucose metabolism in cancer: can it be exploited for cancer therapy? Nat Rev Cancer. 2016;16:635-49.

26. Liberti MV, Dai Z, Wardell SE, Baccile JA, Liu X, Gao X, et al. A predictive model for selective targeting of the warburg effect through GAPDH inhibition with a natural product. Cell Metab. 2017;26:648-59 e648.

27. Perera RM, Bardeesy N. Pancreatic cancer metabolism: breaking it down to build it back up. Cancer Disco. 2015;5:1247-61.

28. Peng L, Ran YL, Hu H, Yu L, Liu Q, Zhou Z, et al. Secreted LOXL2 is a novel therapeutic target that promotes gastric cancer metastasis via the Src/FAK pathway. Carcinogenesis. 2009;30:1660-9.

29. Wong CC, Tse AP, Huang YP, Zhu YT, Chiu DK, Lai RK, et al. Lysyl oxidase-like 2 is critical to tumor microenvironment and metastatic niche formation in hepatocellular carcinoma. Hepatology. 2014;60:1645-58.

30. Ahn SG, Dong SM, Oshima A, Kim WH, Lee HM, Lee SA, et al. LOXL2 expression is associated with invasiveness and negatively influences survival in breast cancer patients. Breast Cancer Res Treat. 2013;141:89-99.

31. Peinado $H$, Moreno-Bueno $G$, Hardisson D, Perez-Gomez E, Santos V, Mendiola M, et al. Lysyl oxidase-like 2 as a new poor prognosis marker of squamous cell carcinomas. Cancer Res. 2008;68:4541-50.

32. Peinado H, Del Carmen Iglesias-de la Cruz M, Olmeda D, Csiszar K, Fong KS, Vega $\mathrm{S}$, et al. A molecular role for lysyl oxidase-like 2 enzyme in snail regulation and tumor progression. EMBO J. 2005;24:3446-58.

33. Canesin G, Cuevas EP, Santos V, Lopez-Menendez C, Moreno-Bueno G, Huang Y, et al. A. Cano, Lysyl oxidase-like 2 (LOXL2) and E47 EMT factor: novel partners in E-cadherin repression and early metastasis colonization. Oncogene. 2015;34:951-64.

34. Salvador F, Martin A, Lopez-Menendez C, Moreno-Bueno G, Santos V, VazquezNaharro A, et al. Lysyl Oxidase-like Protein LOXL2 promotes lung metastasis of breast cancer. Cancer Res. 2017;77:5846-59.

35. Ruckert F, Joensson P, Saeger HD, Grutzmann R, Pilarsky C. Functional analysis of LOXL2 in pancreatic carcinoma. Int J Colorectal Dis. 2010;25:303-11.

36. Park JS, Lee JH, Lee YS, Kim JK, Dong SM, Yoon DS. Emerging role of LOXL2 in the promotion of pancreas cancer metastasis. Oncotarget. 2016;7:42539-52.

37. Tanaka N, Yamada S, Sonohara F, Suenaga M, Hayashi M, Takami H, et al. Clinical implications of Lysyl Oxidase-Like Protein 2 expression in pancreatic cancer. Sci Rep. 2018;8:9846.

38. Jiang $\mathrm{SH}$, Li J, Dong FY, Yang JY, Liu DJ, Yang XM, et al. Increased serotonin signaling contributes to the warburg effect in pancreatic tumor cells under metabolic stress and promotes growth of pancreatic tumors in mice. Gastroenterology. 2017;153:277-91 e219.

39. Denko NC. Hypoxia, HIF1 and glucose metabolism in the solid tumour. Nat Rev Cancer. 2008;8:705-13.

40. Huang LE, Gu J, Schau M, Bunn HF. Regulation of hypoxia-inducible factor 1alpha is mediated by an O2-dependent degradation domain via the ubiquitinproteasome pathway. Proc Natl Acad Sci USA. 1998;95:7987-92.

41. Cebria-Costa JP, Pascual-Reguant L, Gonzalez-Perez A, Serra-Bardenys G, Querol J, Cosin M, et al. LOXL2-mediated H3K4 oxidation reduces chromatin accessibility in triple-negative breast cancer cells. Oncogene. 2020;39:79-121.

42. Almacellas-Rabaiget O, Monaco P, Huertas-Martinez J, Garcia-Monclus S, ChiconBosch M, Maqueda-Marcos S, et al. LOXL2 promotes oncogenic progression in alveolar rhabdomyosarcoma independently of its catalytic activity. Cancer Lett. 2020;474:1-14.

43. Schietke R, Warnecke C, Wacker I, Schodel J, Mole DR, Campean V, et al. The lysyl oxidases LOX and LOXL2 are necessary and sufficient to repress E-cadherin in hypoxia: insights into cellular transformation processes mediated by HIF-1. J Biol Chem. 2010;285:6658-69.

44. Cox TR, Rumney RMH, Schoof EM, Perryman L, Hoye AM, Agrawal A, et al. The hypoxic cancer secretome induces pre-metastatic bone lesions through lysyl oxidase. Nature. 2015;522:106-10.

45. Wong CC, Gilkes DM, Zhang H, Chen J, Wei H, Chaturvedi $\mathrm{P}$, et al. Hypoxiainducible factor 1 is a master regulator of breast cancer metastatic niche formation. Proc Natl Acad Sci USA. 2011;108:16369-74.

46. Pez F, Dayan F, Durivault J, Kaniewski B, Aimond G, Le Provost GS, et al. The HIF-1inducible lysyl oxidase activates HIF-1 via the Akt pathway in a positive regulation loop and synergizes with HIF-1 in promoting tumor cell growth. Cancer Res. 2011;71:1647-57.

47. Wu L, Zhu Y. The function and mechanisms of action of LOXL2 in cancer (Review). Int J Mol Med. 2015;36:1200-4.

48. Cano A, Santamaria PG, Moreno-Bueno G. LOXL2 in epithelial cell plasticity and tumor progression. Future Oncol. 2012;8:1095-108.

49. Millanes-Romero A, Herranz N, Perrera V, Iturbide A, Loubat-Casanovas J, Gil J, et al. Regulation of heterochromatin transcription by Snail1/LOXL2 during epithelial-to-mesenchymal transition. Mol Cell. 2013;52:746-57.
50. Iturbide A, Pascual-Reguant L, Fargas L, Cebria JP, Alsina B, Garcia de Herreros A, et al. LOXL2 Oxidizes Methylated TAF10 and controls TFIID-dependent genes during neural progenitor differentiation. Mol Cell. 2015;58:755-66.

51. Moon HJ, Finney J, Xu L, Moore D, Welch DR, Mure M. MCF-7 cells expressing nuclear associated lysyl oxidase-like 2 (LOXL2) exhibit an epithelial-tomesenchymal transition (EMT) phenotype and are highly invasive in vitro. J Biol Chem. 2013;288:30000-8.

52. Iturbide A, Garcia de Herreros A, Peiro S. A new role for LOX and LOXL2 proteins in transcription regulation. FEBS J. 2015;282:1768-73.

53. Li R, Wang Y, Zhang X, Feng M, Ma J, Li J, et al. Exosome-mediated secretion of LOXL4 promotes hepatocellular carcinoma cell invasion and metastasis. Mol Cancer. 2019;18:18.

54. Jiang SH, Zhu LL, Zhang M, Li RK, Yang Q, Yan JY, et al. GABRP regulates chemokine signalling, macrophage recruitment and tumour progression in pancreatic cancer through tuning $\mathrm{KCNN4} 4$-mediated $\mathrm{Ca}(2+)$ signalling in a GABAindependent manner. Gut. 2019;68:1994-2006.

\section{ACKNOWLEDGEMENTS}

We thank all members of the tumor microenvironment research group of State Key Laboratory of Genes and Related Genes for assistance with this study. This work was supported by grants from the National Natural Science Foundation of China (No. 81902370, No. 81872242, and No. 32170910), the Shanghai Municipal Health Commission (No. 202040104), Shanghai Sailing Program (19YF1445700), Natural Science Foundation of Jiangsu Province (BK20211124), and Zhenjiang Key Research and Development Program (SH2021037).

\section{AUTHOR CONTRIBUTIONS}

$\mathrm{RL}, \mathrm{HL}, \mathrm{LZ}$, and $\mathrm{XZ}$ carried out in vitro cell experiments, manuscript preparation, and statistical analysis; QL and BN carried out in vivo experiments; DL provided clinical specimens and made clinical pathology evaluations; LH performed IHC analysis; ZZ, YZ, XW and SJ conceived, designed, supervised, analyzed and interpreted the study and provided critical review.

\section{ETHICAL APPROVAL}

The research was approved by the Ethics Committee of Shanghai Jiao Tong University Affiliated Ren Ji Hospital.

\section{COMPETING INTERESTS}

The authors declare no competing interests.

\section{ADDITIONAL INFORMATION}

Supplementary information The online version contains supplementary material available at https://doi.org/10.1038/s41419-021-04391-3.

Correspondence and requests for materials should be addressed to Yanli Zhang, Xu Wang or Shu-Heng Jiang.

Reprints and permission information is available at http://www.nature.com/reprints

Publisher's note Springer Nature remains neutral with regard to jurisdictional claims in published maps and institutional affiliations.

Open Access This article is licensed under a Creative Commons Attribution 4.0 International License, which permits use, sharing, adaptation, distribution and reproduction in any medium or format, as long as you give appropriate credit to the original author(s) and the source, provide a link to the Creative Commons license, and indicate if changes were made. The images or other third party material in this article are included in the article's Creative Commons license, unless indicated otherwise in a credit line to the material. If material is not included in the article's Creative Commons license and your intended use is not permitted by statutory regulation or exceeds the permitted use, you will need to obtain permission directly from the copyright holder. To view a copy of this license, visit http://creativecommons. org/licenses/by/4.0/.

(c) The Author(s) 2021 